\title{
Biological applications of kinetics of wetting and spreading
}

G. Ahmed, O. Arjmandi Tash, J. Cook, A. Trybala, V. Starov*

Department of Chemical Engineering, Loughborough University, Loughborough, LE11 3TU, UK

\section{Contents}

Introduction

1. Spreading of Newtonian liquids in the case of non-porous and porous substrates Spreading of Newtonian fluids over non-porous substrates

Spreading of Newtonian fluids over porous substrates

2. Spreading of blood over porous substrates

Blood spreading on porous substrate - complete wetting case

Blood spreading on porous substrate - partial wetting case

3. Simultaneous spreading and evaporation kinetics of blood

Kinetics of evaporation of blood and pattern formation

4. Spreading over hair

Hair and hair wetting properties

Hair products, Aculyns polymeric solutions

Rheology of Aculyns solutions

Spreading of polymeric solutions over hair tress and wetting transition

Foam drainage

Foam drainage on porous materials

\section{Acknowledgements}

Nomenclature

\section{References}

*To whom correspondence should be addressed V.M.Starov@lboro.ac.uk 


\begin{abstract}
Wetting and spreading kinetics of biological fluids has gained a substantial interest recently. The importance of these fluids in our lives has driven the pace of publications. Globally scientists have ever growing interest in understanding wetting phenomena due to its vast applications in biological fluids. It is impractical to review extremely large number of publications in the field of kinetics of complex biological fluids and cosmetic solutions on diverse surfaces. Therefore, biological and cosmetic applications of wetting and spreading dynamics are considered in the following areas: (i) Spreading of Newtonian liquids in the case of non-porous and porous substrates. It is shown that the spreading kinetics of a Newtonian droplet on non-porous and porous substrate can be defined through theoretical relations for droplet base radius on time, which agree well with the experimental results; (ii) Spreading of blood over porous substrates. It is shown that blood, which has a complex nonNewtonian rheology, can be successfully modelled with the help of simple power-law model for shear-thinning non-Newtonian liquids; (iii) Simultaneous spreading and evaporation kinetics of blood. This part enlightens different underlying mechanisms present in the wetting, spreading, evaporation and dried pattern formation of the blood droplets on solid substrates; (iv) Spreading over hair. In this part the wetting of hair tresses by aqueous solutions of two widely used by industry commercially available polymers, AculynTM 22 and AculynTM 33, are discussed. The influence of non-Newtonian rheology of these polymer solutions on the drainage of foams produced from these solutions is also briefly discussed.
\end{abstract}

\title{
Introduction
}

In the last two decades, the interest in wetting and spreading kinetics has increased immensely, particularly since 2010. The rate of increase of publications in the field of wetting and spreading are increasing exponentially due to its vast applications in every field of life. These applications are not limited to naturally occurring processes, but influence industries as well. For example, rain droplets on a window, tears formation in the eyes [1], inkjet printing applications [2], coating, spray painting [3], forensics [4], digital micro-fluidics, pesticide spraying, drug delivery, DNA analysis [5], disease detection [6], cosmetics, etc. The focus is mainly on the biological applications of wetting and spreading, in which biological fluids and cosmetic solutions on biological substrates (hair) is reviewed.

The interaction of these complex liquids with complex surfaces occurs frequently not only in our daily lives but also in different technological applications. Many cosmetics such as skin-care creams and hair-care products are formulated to be applied on skin and hair which are complex surfaces. Complex polymer/surfactant solutions (in the form of droplets or foams) interact with human hair during application of shampoos, conditioners or hair colorants. Recent investigations have confirmed that foams, which are a complex liquid, are an efficient alternative method of drug delivery on the skin of patients, which is a complex porous surface. Although lotions, creams, gels and ointment are the most common topical vehicle delivery systems, foams are delivery systems which grow in popularity in dermatology [7].

Biological fluids are complex liquids, made of different components which interact with each other. These interactions can cause the physical mechanisms already established for simple fluids to change considerably for biological fluids undergoing wetting, spreading, imbibition and evaporation. The most important example of biological fluid is blood, which is a complex colloidal suspension critical 
to human existence, containing proteins, various cells, and plasma. However, it is very important to model the physical mechanisms occurring during wetting phenomena of blood [5].

In all the above-mentioned examples, understanding the interaction between complex liquids and complex surfaces at interfaces at different nano, meso and macro length scales is essential in order to understand physical mechanisms, gain essential stored information, enhance product quality and functionality in multidisciplinary applications. In addition, the interaction condition of complex liquids with complex surfaces is very important because, for example in a number of situations, it can affect the kinetics of delivery of active substances from complex liquids to the target places inside the complex surfaces. In order to make a proper choice of the process parameters and improve the performance of the system, the properties of the complex liquids should be considered in connection with the properties of complex surfaces.

One of the first investigations of the wetting of droplets dates back to early 19th century. Young developed the role of contact angle in wetting dynamics of the droplet [8]. Even a steady state of droplet's geometry is not straightforward: according to Kelvin's equation the droplet can be at equilibrium with oversaturated vapour only, which is usually not the case. Hence, all deposited droplets are in the state of simultaneous spreading/evaporation. The latter means, if all processes of wetting, spreading, imbibition and evaporation are taken into account, then the resulting problem becomes a very complicated one that has extensively been studied by researchers and scientists worldwide. Nature of fluids, substrates, surrounding environment and involved physics are vital factors influencing the intricacy of the problem.

Nature of fluid and fluid rheology is vital to wetting phenomena. Fluids can have Newtonian or nonNewtonian behaviour (viscosity varying with shear rate). Spreading of Newtonian droplets on solid substrates is discussed in Part 1. It was proven that introducing surface forces allows describing complete spreading of Newtonian fluid droplet on solid substrates in the case of complete wetting. Spreading law in the case of completely wetting Newtonian fluids over porous substrates is developed and two spreading regimes are identified. Theories presented in this section show a good agreement with available experimental data.

In the next section, Part 2, it is shown that blood is a complex fluid, which however, can be modelled with the help of simple model of a power-law non-Newtonian shear thinning fluid. Experimental results of measurement of blood's viscosity with varying shear rates justify its power-law shearthinning behaviour. Based on power-law model a theory is developed to account for blood spreading/imbibition over porous substrates. Predicted results from the theoretical model have a reasonable agreement with experimental measurements.

Blood droplets as all droplets are susceptible to drying by evaporation after deposition. Dried patterns formed by blood droplets have drawn increasing attention of scientists as these patterns carry a lot of information, especially from both healthcare and forensics perspective. In Part 3, spreading and evaporation kinetics of the dried blood samples are reviewed. Factors that affect the patterns formed by the dried blood samples are discussed.

Finally in Part 4 recent results on wetting and spreading of complex cosmetic polymeric solutions over hair tresses are presented, where wetting transition was found in some cases. Foams generated from these cosmetic solutions are also investigated in the case of free foam drainage. The effect of non-Newtonian rheology of these solutions on the rate of liquid release from foam is discussed. In 
addition, the recently proposed theory of foam drainage placed on top of a porous substrate is briefly explained.

\section{Spreading of Newtonian liquids in the case of non-porous and porous substrates}

Wetting and spreading of Newtonian fluids on non-porous as well as porous surfaces occur widely in nature and these processes are significant to many biological and bio-industrial applications. Pesticide droplet deposition on plant leaves, movement of water inside plants, DNA microarrays (spreading of DNA in patterned micro-channels), bio-surfactants preventing oil from spreading, blood spreading in blood pattern analysis, spreading of a fluid occurs as tears form thin films [1], are to name only a few biological applications of fluid spreading occurring in our surroundings.

A sessile droplet on a substrate involves three phases: solid, liquid and vapour. The line where all the three phases come into contact is referred to as the apparent three phase contact line. Contact angle is defined as the angle which the solid-liquid interface makes with the liquid-vapour interface. Contact angle defines the wetting property of the substrate for the particular fluid under investigation. Factors affecting the contact angle include surface roughness, chemical composition, temperature, humidity, porosity, etc.

Substrates are not generally homogeneous: there can be topographical imperfections (microscopic, i.e. roughness [9] and macroscopic, i.e. topography [10]) and chemical heterogeneities [11]. Substrates can be porous and spreading kinetics is also largely influenced by absorptive properties of the substrate.

Fluid rheology is important for both wetting and spreading kinetics. Fluids can show Newtonian or non-Newtonian behaviour. Newtonian fluids are fluids that exhibit a directly proportional relationship between the stress and strain. The behaviour of non-Newtonian fluids can be broadly divided into two categories: shear-thinning and shear-thickening. A number of mathematical models for fluid rheology have been suggested. Below it is concentrated on Ostwald de Waele model also known as power-law shear-thinning model, because a wide range of rheological properties of biological fluids can be approximated using this model, and the most important is blood.

Contact line motion has a complex dynamics which is still to be understood even in the case of Newtonian liquids. Due to friction the velocity at the liquid/solid interface should be zero (no-slip condition), but the droplet is in motion along the apparent three phase contact line. This results in artificial singularity along the three phase contact line. Consideration of disjoining pressure acting in the vicinity of the three phase contact line removes the artificial singularity [12]. In the next sections, theoretical models and the current state of investigation of the spreading of Newtonian and nonNewtonian fluid droplets over both non-porous and porous substrates is reviewed.

\section{Spreading of Newtonian fluids over non-porous substrates}

Surface forces in the vicinity of the contact line play a vital role in the kinetics of spreading [12]. Disjoining/conjoining pressure action determines both equilibrium and kinetics of spreading. Action of disjoining/conjoining pressure in the vicinity of the apparent three phase contact line results in the following phenomenon: the liquid profile does not meet the solid substrate at a particular point, but in close proximity to the substrate the thickness of the fluid tends to the thickness determined by the vapour pressure in the ambient air (Fig.1). The existence of this thin film has been experimentally proven earlier $[12,13]$. Considerable literature (experimental, theoretical, and numerical) is available on droplet spreading over solid substrates [1,9,13-15]. 


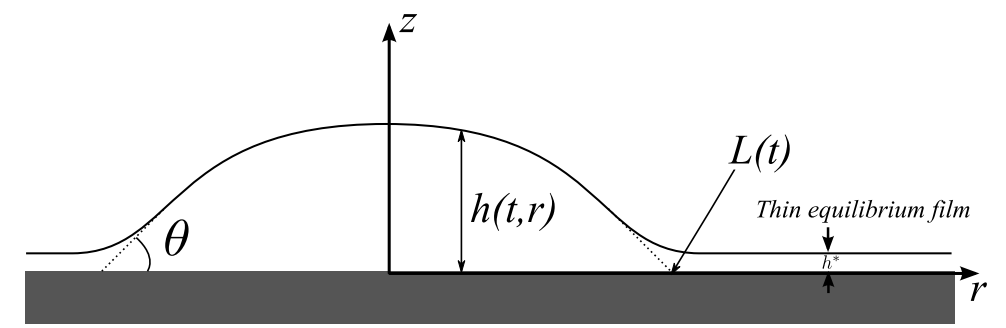

Fig. 1: Spreading of liquid droplet over flat solid substrate in the vicinity of the apparent three-phase contact line. $\mathrm{L}(\mathrm{t})$ - effective radius of spreading; thin liquid film is determined by vapour adsorption from the ambient air.

Different stages of spreading regimes develop in the course of spreading: capillary and gravitational regimes are the most important. Even in the case of spreading of small droplets the final stage of spreading becomes gravitational. Transition time, $t_{c}$, to go from capillary regime to the gravitational regime of spreading takes place when the radius of spreading, $L(t)$, becomes big enough (Fig.1):

$$
L\left(t_{c}\right) \sim a=\sqrt{\frac{\gamma}{\rho g}},
$$

where $L(t)$ is the base radius of the droplet, $\gamma$ is the liquid/air interfacial tension, $\rho$ is the density of the fluid, $a$ is the capillary length, $t_{c}$ is the time taken for the capillary regime of spreading and $g$ is the gravitational acceleration. Profile of the axisymmetric droplet spreading on a non-porous substrate is governed by the following equation [12]:

$$
\frac{\partial h}{\partial t}=\frac{1}{3 \mu r} \frac{\partial}{\partial r}\left(r h^{3} \frac{\partial p}{\partial r}\right),
$$

where $h(t, r)$ is the liquid profile, $r$ is the radial co-ordinate, $p(t, r)$ is the pressure, and $\mu$ is the viscosity of fluid.

$$
p=p_{a}-\gamma \frac{1}{r} \frac{\partial}{\partial r}\left(r \frac{\partial h}{\partial r}\right)-\Pi(h)+\rho g h,
$$

where $p_{a}$ is the ambient air pressure, and $\Pi(h)$ is the disjoining pressure [12]. $p$ varies according to the thickness of the droplet [12],

$$
p(t, r)=\left\{\begin{array}{ll}
p_{a}-\gamma \frac{1}{r} \frac{\partial}{\partial r}\left(r \frac{\partial h}{\partial r}\right)+\rho g h & h \geq 10^{-5} \mathrm{~cm} \\
p_{a}-\gamma \frac{1}{r} \frac{\partial}{\partial r}\left(r \frac{\partial h}{\partial r}\right)-\Pi(h) & h \leq 10^{-5} \mathrm{~cm}
\end{array} .\right.
$$

In the case of non-volatile the liquid volume, $V$, remains constant. For small droplets, with radius smaller than capillary length the pressure in the droplet after the initial inertial stage in the capillary regime is given by,

$$
p=p_{a}-\gamma \frac{1}{r} \frac{\partial}{\partial r}\left(r \frac{\partial h}{\partial r}\right) .
$$

So, Eq. (2) in the capillary regime takes the form,

$$
\frac{\partial h}{\partial t}=\frac{\gamma}{3 \mu r} \frac{\partial}{\partial r}\left\{r h^{3} \frac{\partial}{\partial r}\left[\frac{1}{r} \frac{\partial}{\partial r}\left(r \frac{\partial h}{\partial r}\right)\right]\right\} .
$$


Therefore, the boundary conditions at $r=0, \frac{\partial h}{\partial r}=\frac{\partial^{3} h}{\partial r^{3}}=0$. Spreading law with the help of similarity solutions [12] can then be written as,

$$
L(t)=0.65 C\left(\frac{\gamma V^{3}}{\mu}\right)^{0.1} t^{0.1}
$$

where $C$ is an integration constant. This results in the spreading rate, $U(t)$, is given by,

$$
U(t)=\frac{d L(t)}{d t}=0.65 C\left(\frac{\gamma V^{3}}{\mu}\right)^{0.1} t^{0.9}
$$

which is equivalent to famous Tanner's law of spreading for complete wetting $[12,16]$. In the gravitational spreading regime, the radius of spreading is greater than capillary length; capillary pressure can be neglected, hence:

Therefore equation (2) becomes:

$$
p=p_{a}+\rho g h
$$

$$
\frac{\partial h}{\partial t}=\frac{\rho g}{3 \mu r} \frac{\partial}{\partial r}\left\{r h^{3} \frac{\partial h}{\partial r}\right\}
$$

Spreading law in the gravitational regime with the help of similarity solutions [12] can be written as,

$$
L(t)=0.78\left(\frac{\rho g V^{3}}{\mu}\right)^{1 / 8} t^{1 / 8}
$$

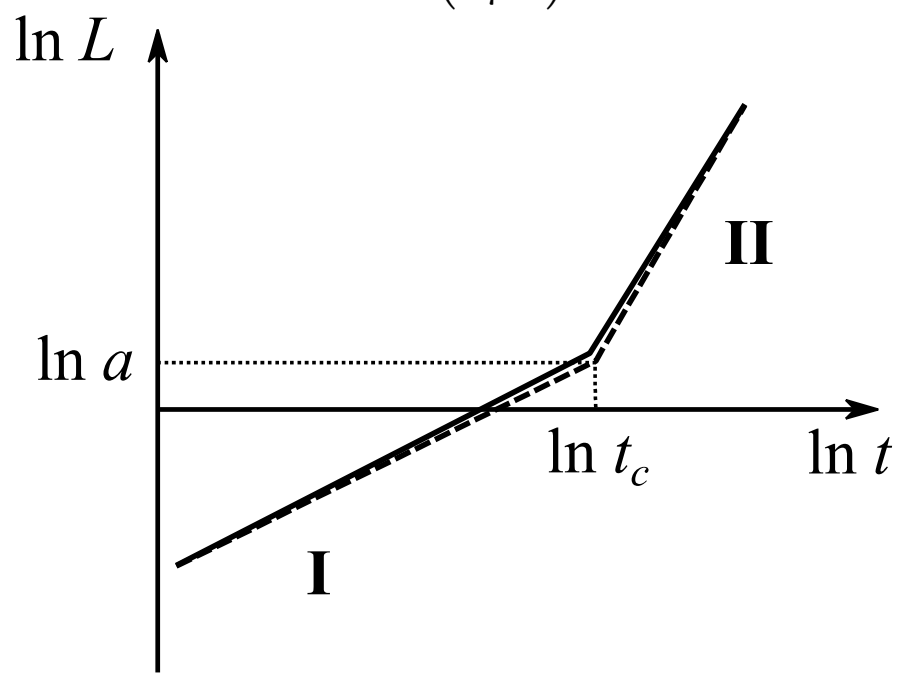

Fig. 2: Log-log plot showing the variation of the spreading radius with time. (I) capillary spreading regime; (II) gravitational spreading regime. Reproduced with permission from Ref. [12]. Copyright (C) 2007 CRC Press.

Capillary spreading regime switches to gravitational spreading regime at capillary length, $a$ and time, $t_{c}$, see Fig. 2.

As mentioned earlier, influence of surface forces on the droplet spreading is described with the help of disjoining pressure. In the case of complete wetting, equation for Newtonian, non-volatile fluid droplet on a smooth, homogeneous solid substrate according to [12,17] is as follows:

$$
\frac{\partial h}{\partial t}=-\frac{\gamma}{3 \mu r} \frac{\partial}{\partial r}\left\{r h^{3} \frac{\partial}{\partial r}\left[\frac{1}{r} \frac{\partial}{\partial r}\left(r \frac{\partial h}{\partial r}\right)\right]-\frac{n A}{\gamma h^{n+1}} \frac{\partial h}{\partial r}\right\},
$$


where $\Pi(h)=A / h^{n}, \quad n=2$ or 3 (in the case $n=3, A$ is the Hamaker constant). With gravitational and inertial effects negligible spreading radius dependence on time is given by $[12,17]$ in this case by exactly the same dependency as (7) but in this case the integration constant can be calculated and the result is:

$$
L_{0}(t)=0.89\left(\frac{\gamma V^{3}}{\mu}\right)^{0.1} t^{0.1}
$$

Chen et al. have conducted a series of experiments of polydimethylsiloxane droplet spreading on a glass substrate $[17,18]$. Experiments satisfied the conditions of applicability of the theory developed above and were conducted at room temperature. Results of comparison of the experiments (point) with theoretical dependences (solid lines) are shown in Fig. 3.

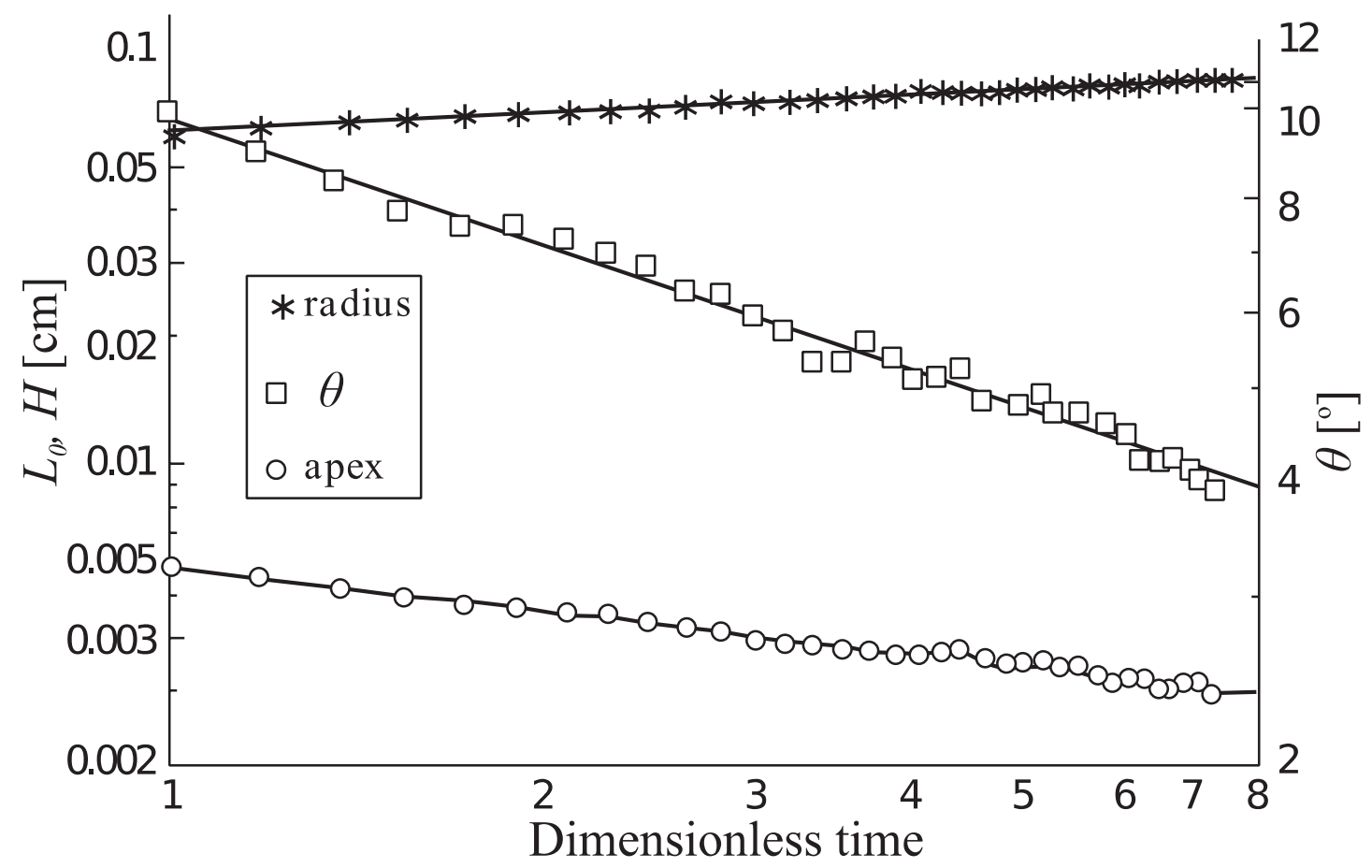

Fig. 3. Comparison of experimental results for radius of spreading, $L$, apex, $H$, and dynamic contact angle, $\theta$, with theory predictions (solid lines) [5]. Reproduced with permission from Ref. [12]. Copyright (C) 2007 CRC Press.

Theory presented in [12] proved that introducing surface forces allow to describe completely spreading of Newtonian fluid droplet on solid substrates in the case of complete wetting, see Fig. 4. 


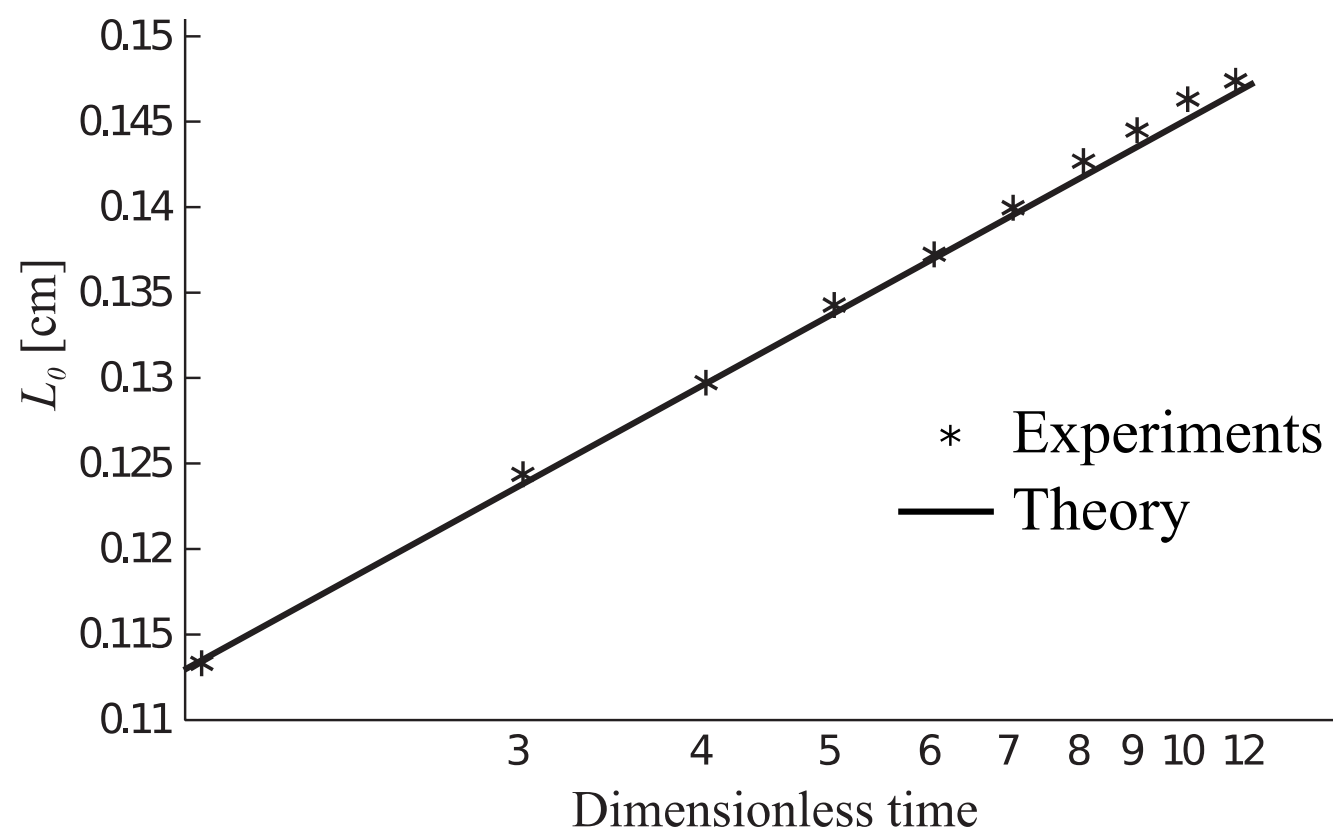

Fig. 4: Comparison of the experimental data with the theory (equation (13)) shows an excellent agreement for the radius of the spreading droplet [17]. Reproduced with permission from Ref. [17]. Copyright $(1994$ Elsevier B.V.

\section{Spreading of Newtonian fluids over porous substrates}

The substrates are not always smooth, homogeneous and non-porous. Substrates can be rough and even porous. Fluid wetting/spreading on permeable/porous substrates is considerably different process as compared with wetting/spreading on homogeneous/solid substrates $[12,19,20]$. Due to vast applications, spreading dynamics in/over porous medium has received significant attention [12,15,2025].

In the course of spreading over porous substrates for the case of small capillary numbers droplets on a porous substrate maintained a spherical shape [12,20,24,26,27]. Theoretical description of spreading of fluid droplets over porous substrates is frequently based on an ad hoc slippage condition [21,24]. However, rather than using slippage condition Brinkman's equation is used to describe the fluid flow inside the porous medium [12,20,26,27]. Theory briefly presented below is valid for complete wetting and incorporates lubrication approximation [20].

The thickness of the porous layer, $h_{s}$, is assumed to be much smaller than the drop height, that is, $h_{s} \ll h^{*}$, where $h^{*}$ is the characteristic scale of the droplet height. The droplet profile is assumed to have a low slope (lubrication approximation) and the influence of the gravity is neglected (small drops, Bond number much less than 1). That is, only capillary forces are taken into consideration. A system of two differential equations have been deduced to describe the time dependency of the radius of the droplet base, $L(t)$, and the wetted region in the porous medium, $l(t)$, with time (see Fig. 5): 


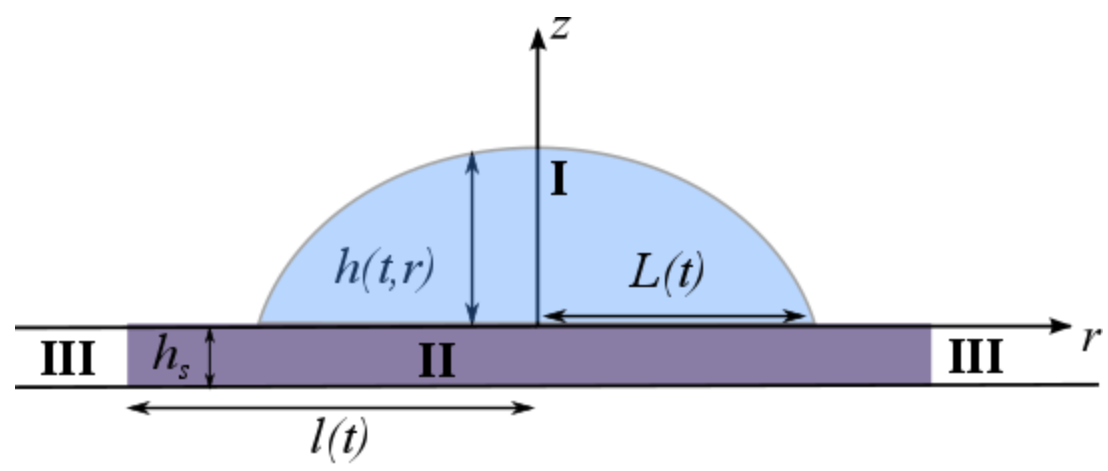

Fig. 5. Schematic of the droplet on a porous substrate with thickness, $h_{s}$; I - fluid droplet, II - wetted region in the porous substrate, III - dry region in the porous substrate.

$$
\begin{gathered}
\frac{d L}{d t}=\frac{0.1}{\left(t+t_{0}\right)^{0.9}}\left(\frac{4\left(V_{0}-\pi m h_{s} l^{2}\right)}{\pi}\right)^{0.3}\left(\frac{10 \gamma w}{\mu}\right)^{0.1}+\frac{2 \pi m h_{s} K_{p} p_{c} L / \mu}{3\left(V_{0}-\pi m h_{s} l^{2}\right) \ln \frac{l}{L}}, \\
\frac{d l}{d t}=\frac{K_{p} p_{c}}{\mu l \ln \frac{l}{L}}
\end{gathered}
$$

where $V_{0}$ is initial volume of the droplet, $m$ is the porosity of the porous substrate, $w \sim 0.01$ is the effective lubrication parameter [12]; $K_{p}$ and $p_{c}$ are the permeability of the porous layer and capillary pressure on the moving front inside the porous substrate; $\gamma$ and $\mu$ are the liquid/air interfacial tension and the dynamic viscosity, respectively. These equations are made dimensionless using the following scaling: $\bar{L}=L(t) / L_{m}, \bar{l}=l(t) / l^{*}, \bar{\theta}=\theta(t) / \theta_{m}$ and $\bar{t}=t / t^{*}$, where $L_{m}$ is the maximum radius of droplet base, which is reached at the time instant $t_{m}, l^{*}$ is the radius of wetted region at the end of the process, $\theta_{m}$ is the contact angle at maximum spreading radius, and $t^{*}$ is the time when the imbibition is completed.

Experiments on spreading over porous substrates to verify the suggested theory were performed using silicone oil droplets of different viscosities on cellulose nitrate membranes with different pore sizes [12]. Detailed experimental setup is explained in [20]. Results of the experiments revealed that the spreading can be subdivided into two stages, a fast first stage when the droplet spreads to a maximum radius, $L_{m}$. During the first stage an imbibition front inside the membrane expands only slightly ahead of the spreading drop. After that during the second stage the drop base starts to shrink slowly and the imbibition front expands until the drop completely disappears. Droplet remained spherical shape during both stages of spreading/imbibition. The edge of the wetted region inside the porous layers was always circular and no deviations from the cylindrical symmetry or instabilities were detected. Fig. 6 shows that all the experimental data fell on two universal curves determined according to equations (14) and (15). 


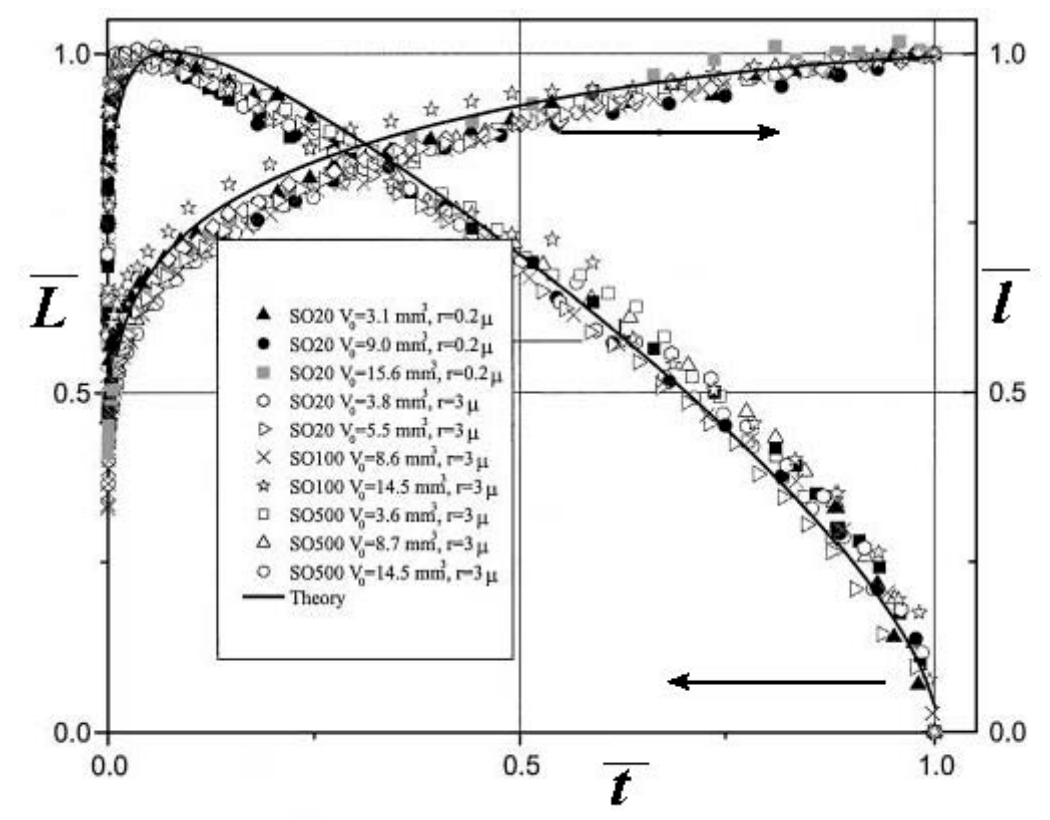

Fig. 6. Dependency of dimensionless droplet base radius, $\bar{L}$, and radius of wetted region inside the porous substrate, $\bar{l}$, on dimensionless time, $\bar{t}$ for experiments and the theory (solid lines) developed in $[12,20]$. Reproduced with permission from Ref. [20]. Copyright (C) 2002 Elsevier B.V.

\section{Spreading of blood over porous substrates}

Investigation of biological fluids with regards to rheology, wetting, spreading, evaporation, drying, and imbibition into different substrates have gained a considerable interest recently. Most important biological fluid is blood, which is a critical for both animal and human survival. Blood is a complex heterogeneous multiphase mixture, made up of red blood cells (RBCs), as well as white blood cells (WBCs) and platelets, all suspended in liquid plasma (also known as blood serum). The haematocrit level (Ht) is defined as the ratio of red blood cells volume to whole blood volume. Blood rheology is highly affected by the haematocrit level [28-30]. RBCs possess elastic deformability and this accounts for blood's complex rheological behaviour [31,32]. For the purpose of wetting/spreading blood can be characterized as non-Newtonian fluid with shear-thinning behaviour [33-35]. The interest in blood's wetting and spreading kinetics has been primarily driven due to the numerous applications of blood that are crucial to our existence, DNA analysis, diagnosis of diseases, medical examination, drug delivery, drug screening, and even blood pattern analysis for forensics investigations [36].

In the previous section spreading and wetting of Newtonian fluids over porous substrate was discussed. Below recent results are presented on wetting and spreading of blood droplets over dry thin porous substrates $[30,37,38]$. The mechanism used for acquiring data for wetting and spreading of blood on porous substrate is a similar to that used at Dried Blood Spot (DBS) sampling technique by National Health Service (NHS) in UK. This technique forms the basis of experiments and modelling method used below, and involves simultaneous spreading and imbibition of blood into the porous substrate. This technique is widely used for blood collection, transportation and storage by NHS. In DBS sampling the blood from syringe/fingertip imbibes into thin porous substrate. Substrate can be filter paper, cotton/cellulose fibres and/or polymer membranes. Blood droplet spreading kinetics over thin porous substrate for the partial and complete wetting cases is considered below using a mathematical model developed under lubrication approximation assumption [37,38]. 


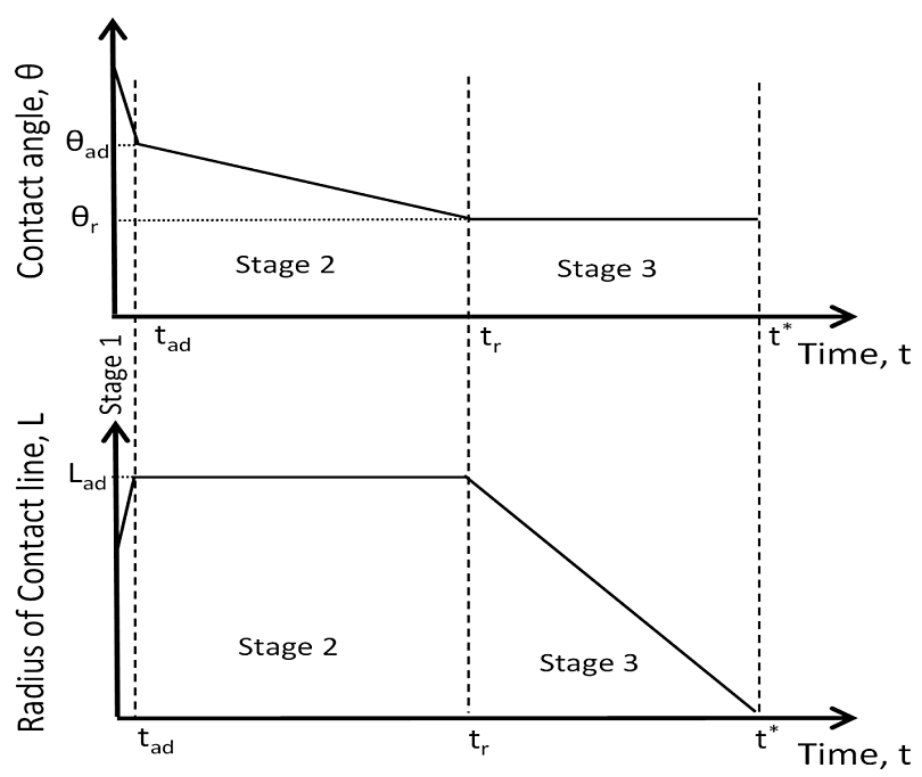

(a)

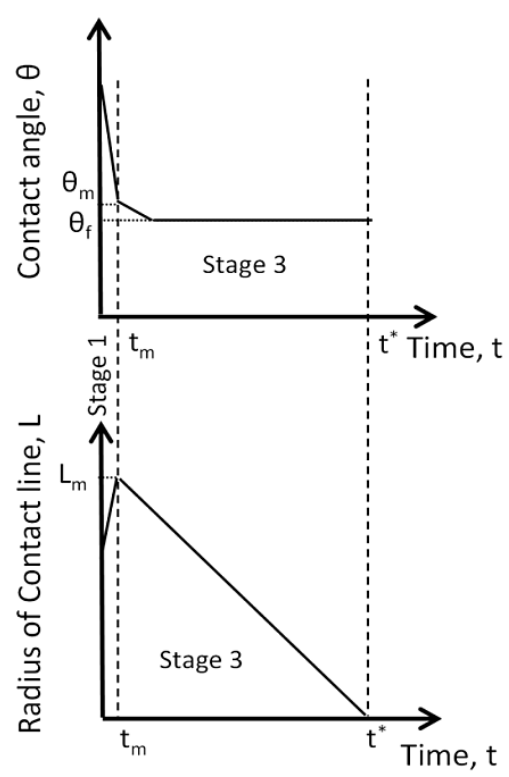

(b)

Fig. 7. Schematics of droplet spreading/imbibition over porous substrate a) partial wetting case: three stages. $L_{a d}$ is the maximum radius of droplet base, $\theta_{a d}$ is the advancing contact angle, $t_{a d}$ is the time when $\theta_{a d}$ is reached, $\theta_{r}$ is the receding contact angle, $t_{r}$ is the time when $\theta_{r}$ is reached and $t^{*}$ is the time when imbibition is finished; b) Complete wetting case: only two stages. $L_{m}$ is the maximum radius of droplet base, $t_{m}$ is the time when $L_{m}$ is reached, $\theta_{m}$ is the contact angle at $t_{m}, t^{*}$ is the time when complete imbibition is finished and $\theta_{f}$ is the final contact angle at the moment $t^{*}$. Note, in the case of complete wetting the stage 2 is absent. Reproduced with permission from Ref.[37]. Copyright (c) 2015 Elsevier B.V.

\section{Blood spreading on porous substrate - complete wetting case}

For experiments blood samples with different haematocrit levels were prepared. Droplets of known volumes of these samples were deposited on thin porous substrates (of known thickness): nitrocellulose membranes, unprocessed, and salinized commercial Whatman 903 filter paper. Details on the experimental setup are described in References [30,37,38]. Results of some experiments are shown in Fig. 8 (for $L(t)$ ) and Fig. 9 (for $l(t)$ (see Fig. 5)). Spreading kinetics of all droplets (Fig. 8) of the droplets is following the case of complete wetting (Fig. 7b). It is shown in Fig. 9 that the concentration of red blood cells affects the size of wetted region inside the porous medium and it differs from that in the case of pure water droplets. Results presented in Fig. 8 confirm that the spreading of blood over Whatman 903 filter paper corresponds to the complete wetting, see Fig. 7. 


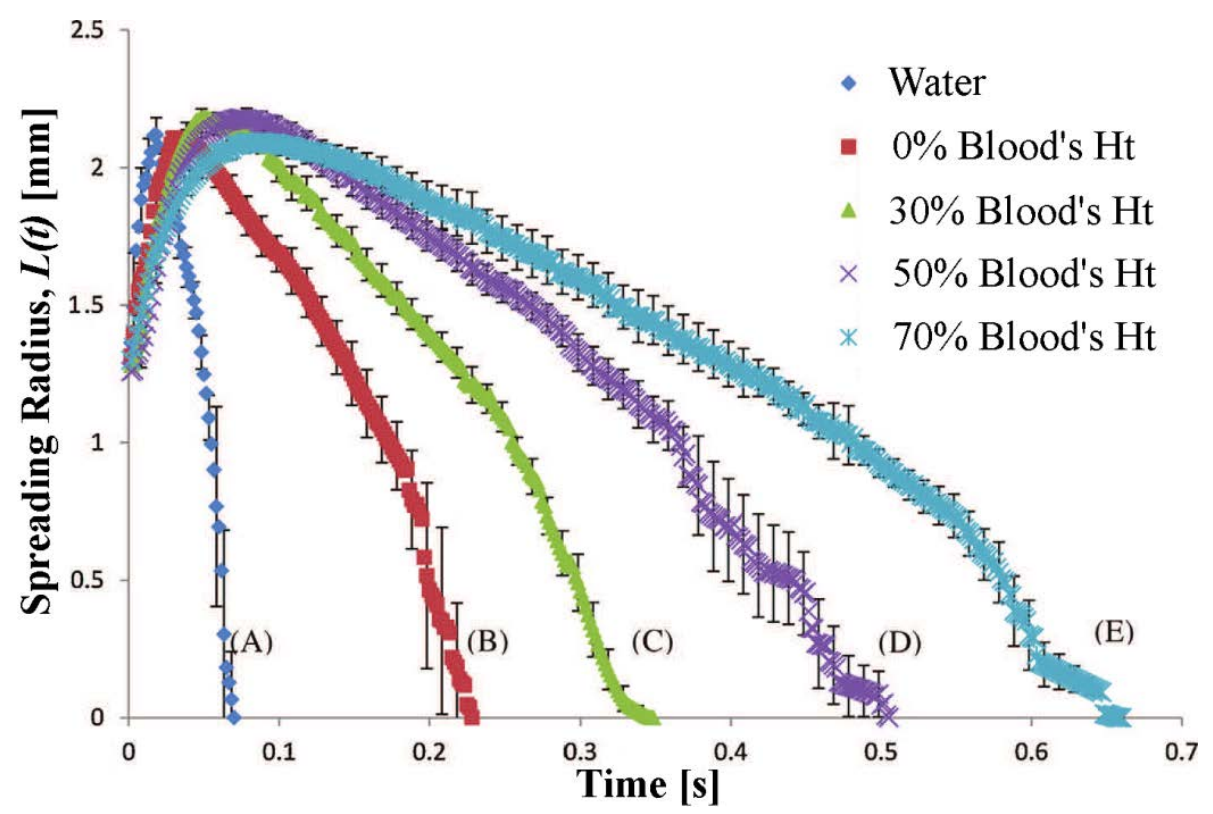

Fig. 8: Experimental results for variation of droplet base radius with time on Whatman 903 filter paper for droplets of (A) $10 \mu \mathrm{l}$ water, (B) $10 \mu \mathrm{l} 0 \%$ Blood Ht, (C) $10 \mu \mathrm{l} \mathrm{30 \%} \mathrm{Blood} \mathrm{Ht,} \mathrm{(D)} 10 \mu \mathrm{l} 50 \%$ Blood Ht, and (E) $10 \mu \mathrm{l} 70 \%$ Blood Ht, where Ht is Haematocrit. Reproduced with permission from Ref. [30]. Copyright (C) 2014 Elsevier B.V.

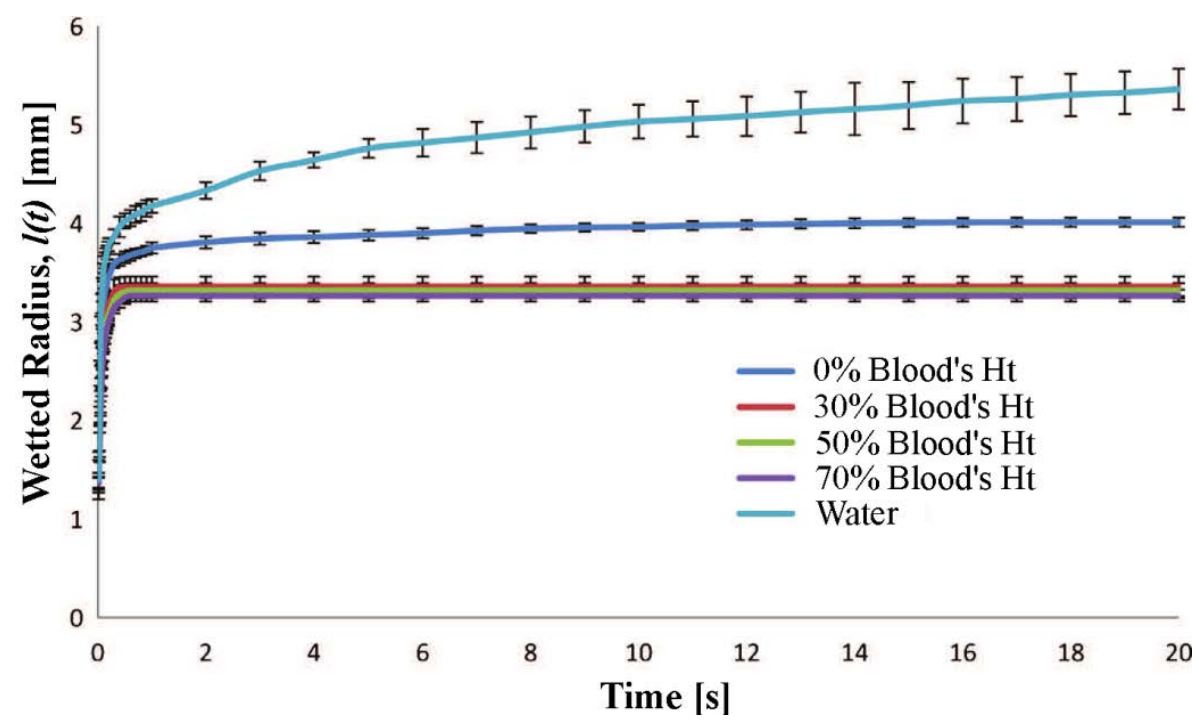

Fig. 9: Experimental results for variation of wetted radius with time for different blood haematocrit levels and pure water on Whatman 903 filter paper, Reproduced with permission from Ref. [30]. Copyright (C) 2014 Elsevier B.V.

Blood at various haematocrit values is a fluid that can be modelled as shear-thinning power law nonNewtonian liquid $[37,38]$. It was demonstrated in $[37,38]$ that the blood with different haematocrit values can be described by Otswal-de Waele power law relationship, $\mu=K \dot{\gamma}^{n-1}$, where $K$ is the flow consistency factor, $\dot{\gamma}$ is the shear rate and $n$ is the flow behaviour index, see Fig. 10 and Table 1 . The flow behaviour index is also referred to as the power-law exponent, which dictates the rheology of the fluid, for $n<1$ shear-thinning fluid, for $n=1$ newtonian fluid and for $n>1$ shear-thickening fluid. 
Table 1: Values of power-law constants $K$ and $n$, for different blood Haematocrit levels. Reproduced with permission from Ref. [30]

\begin{tabular}{|c|c|c|}
\hline Blood Haematocrit level (\%) & $\boldsymbol{K}$ & $\boldsymbol{n}$ \\
\hline 0 & 23.99 & 0.368 \\
\hline 30 & 57.72 & 0.394 \\
\hline 50 & 120.60 & 0.325 \\
\hline 70 & 325.11 & 0.240 \\
\hline
\end{tabular}

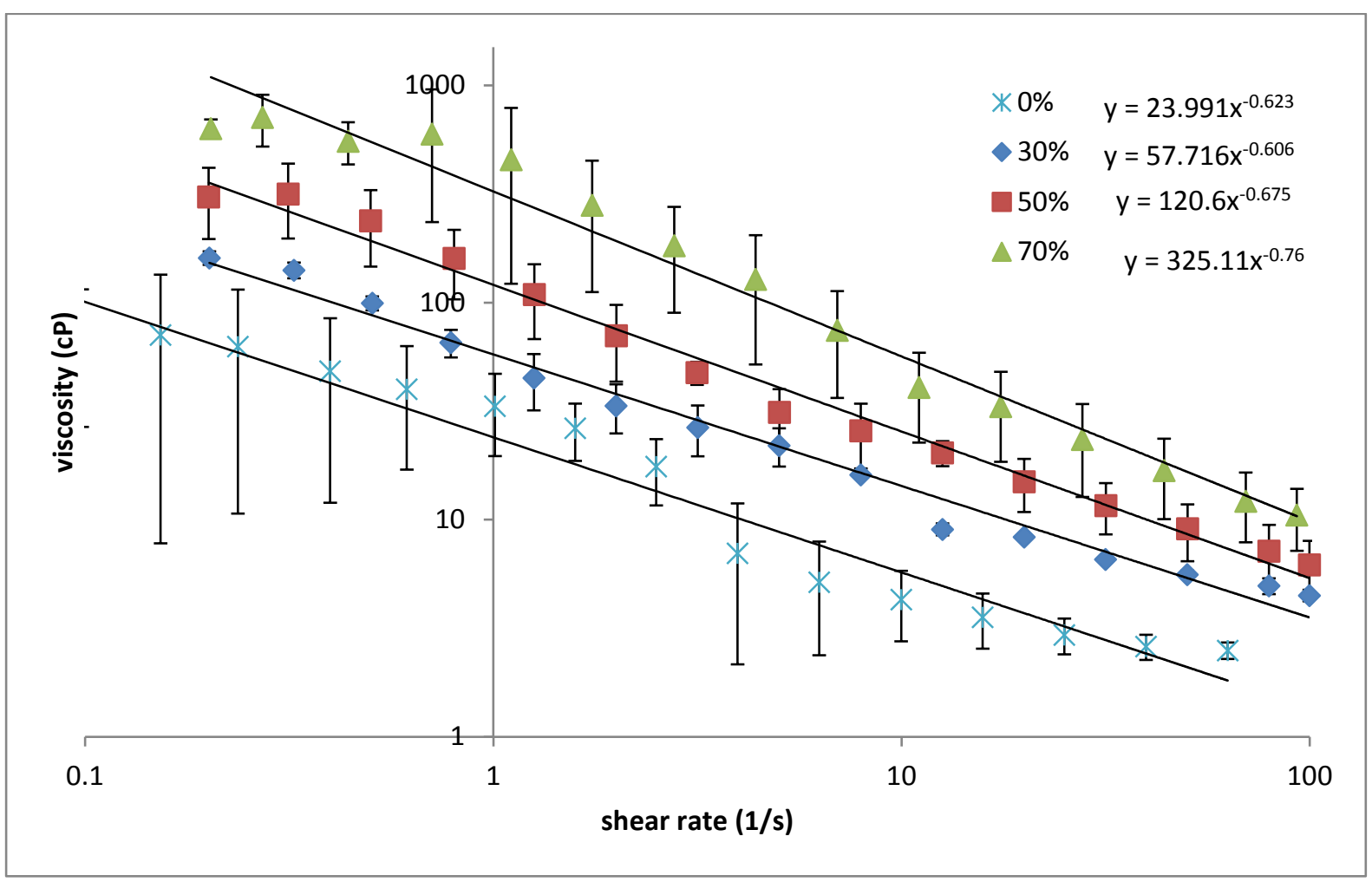

Fig. 10. Power-law dependence of blood's viscosity for different haematocrit levels. Experimental dependences of log Viscosity against log Shear rate. Fitted according to $\mu=K \dot{\gamma}^{n-1}$ dependency. Reproduced with permission from Ref. [30]. Copyright (c) 2014 Elsevier B.V.

The mathematical model developed includes a system of two differential equations, one for droplet base radius variation with time, $L(t)$, and other for evaluating the wetted region inside porous substrate, $l(t)$, as seen in the diagram in the previous section in Fig. 5. Differential equations for $L(t)$ and $l(t)$ of non-Newtonian rheology fluid droplets in the complete wetting case are given as [37,38]:

$$
\begin{gathered}
\frac{d L}{d t}=\frac{\alpha_{n}\left(\frac{V}{V_{0}}\right)^{\frac{1}{3}}\left(\frac{n}{2 n+1} \frac{\lambda}{\alpha_{n}}\left(\frac{\gamma}{K}\right)^{1 / n}\left(\frac{V_{0}}{2 \pi}\right)^{(n+2) / n}\right)^{\alpha_{n}}}{\left(t+t_{0}\right)^{1-\alpha_{n}}} \\
-\frac{2 \pi m h_{s} K_{p} L}{3\left(V_{0}-\pi m h_{s} l^{2}\right)}\left[\frac{(1-n) p_{c}}{K\left(l^{1-n}-L^{1-n}\right)}\right]^{1 / n}, \\
\frac{d l}{d t}=\frac{K p}{l}\left[\frac{(1-n) p_{c}}{K\left(l^{1-n}-L^{1-n}\right)}\right]^{1 / n},
\end{gathered}
$$


where $\alpha_{n}=\frac{n}{3 n+7}, V$ is the volume of the droplet. Detailed derivation of these equations is discussed in [37]. Equations (16),(17) are made dimensionless using the following scaling: $\bar{L}=L(t) / L_{m}, \bar{l}=$ $l(t) / l^{*}, \bar{\theta}=\theta(t) / \theta_{m}$ and $\bar{t}=t / t^{*}$, where $L_{m}$ is the maximum radius of droplet base which is reached at the time instant $t_{m}, l^{*}$ is the radius of wetted region at the end of the process, $\theta_{m}$ is the contact angle at maximum spreading radius, and $t^{*}$ is the time when complete imbibition is finished, that is, the same dimensionless variables as used as in the previous section are used here to dimensionalize the above equations. Fig. 11-13 show comparison of the experimental results with the theoretical model for different blood sample having different Haematocrit levels (hence, different values of $n$, and $K$. These figures demonstrate that the spreading kinetics on porous substrate in the case of complete wetting is governed by two stages as stated earlier in the previous section, i.e. a fast initial stage followed by slow droplet contraction due to loss of droplet volume to the porous substrate.

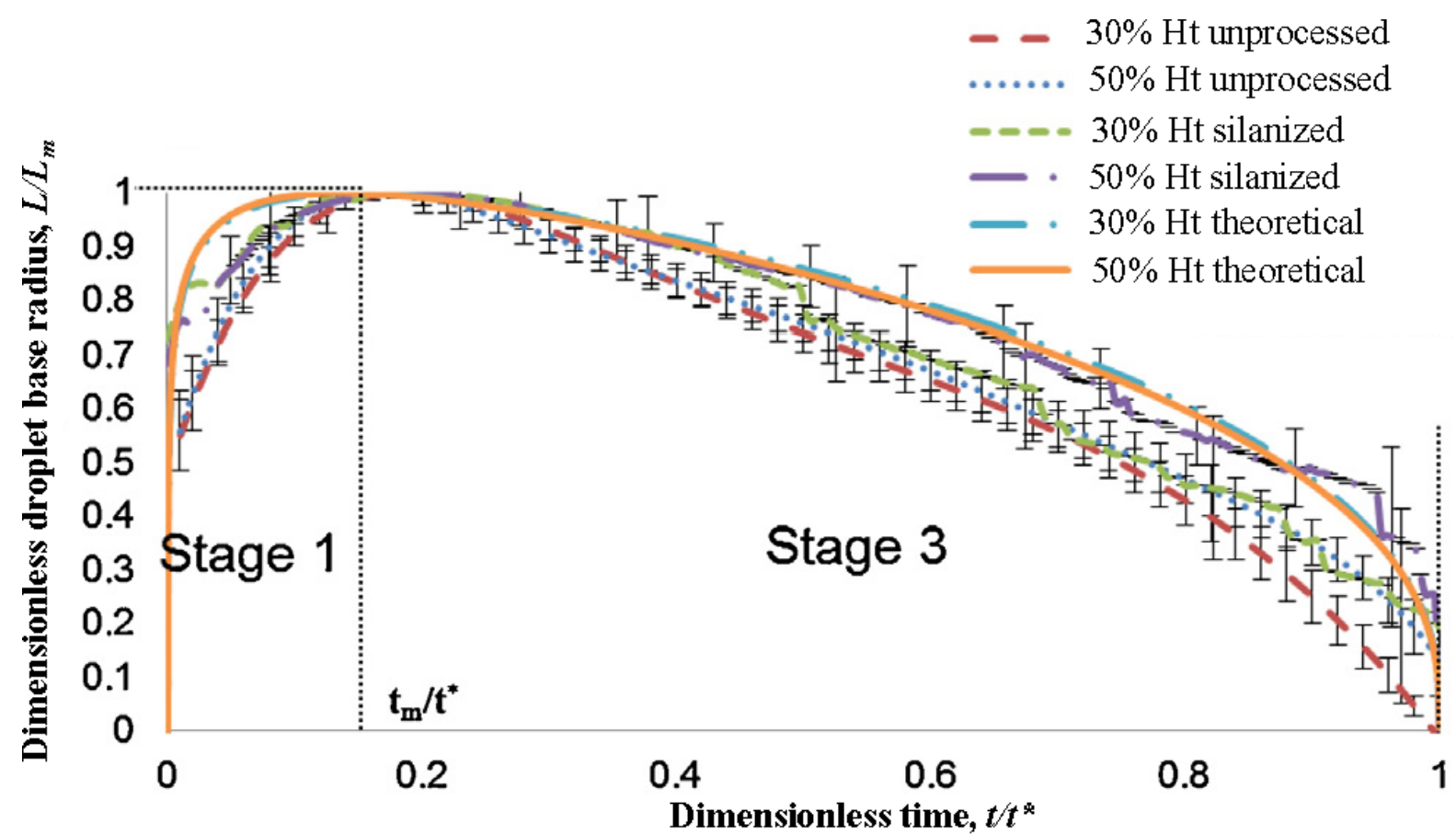

Fig. 11: Comparison between theoretical model and experimental results of evolution of dimensionless droplet base radius on dimensionless time for the case of complete wetting of blood samples on silanized and unprocessed Whatman 903 paper. Reproduced with permission from Ref. [38]. Copyright @ 2016 Elsevier B.V. 


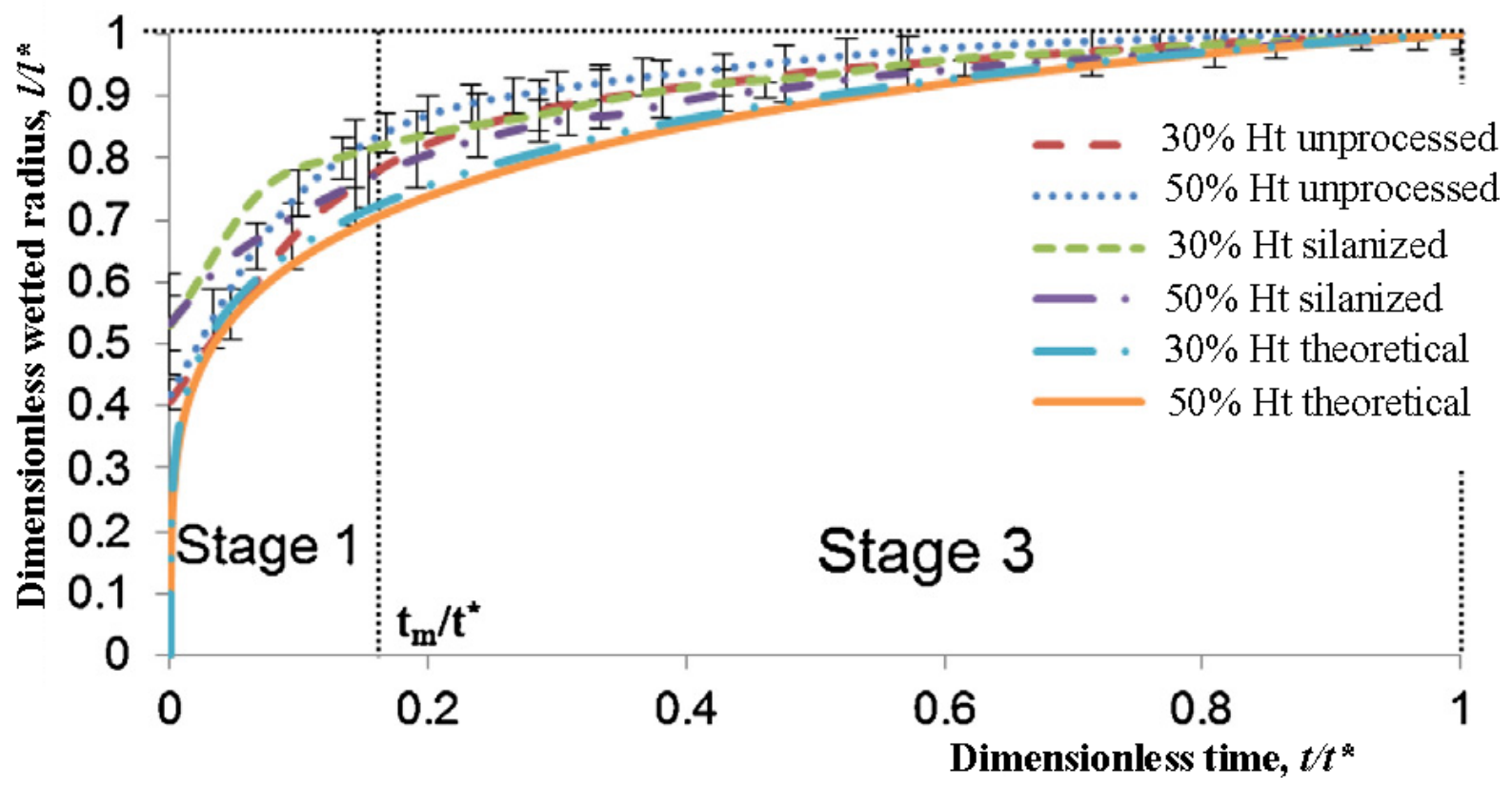

Fig. 12: Comparison between theoretical model and experimental results of evolution of dimensionless wetted radius inside porous substrate on dimensionless time for the case of complete wetting of blood samples on silanized and unprocessed Whatman 903 paper. Reproduced with permission from Ref. [38]. Copyright (C) 2016 Elsevier B.V.

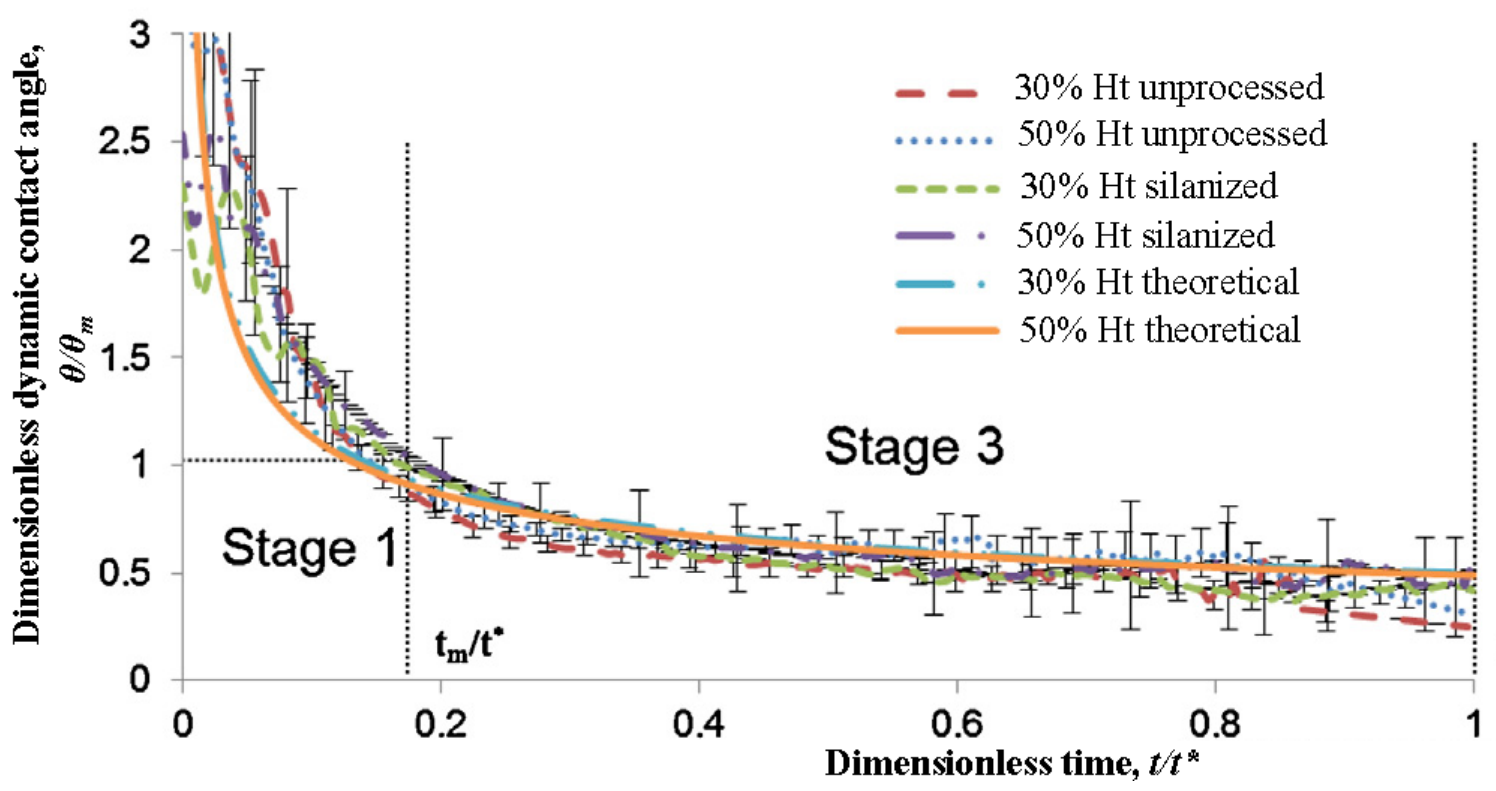

Fig. 13: Comparison between theoretical model and experimental results of evolution of dimensionless dynamic contact angle on dimensionless time for the case of complete wetting of blood samples on silanized and unprocessed Whatman 903 paper. Reproduced with permission from Ref. [38]. Copyright (C) 2016 Elsevier B.V. 


\section{Blood spreading on porous substrate - partial wetting case}

Spreading of droplets over porous substrate in the case of partial wetting can be divided into three stages (Fig. 7). First spreading stage: a fast spreading of the droplet to a maximum base radius value causing the contact angle to decrease to static advancing contact angle and the droplet base to maximum value (Fig. 7). Second stage: radius of the droplet base remained constant while the contact angle reduced from static advancing contact angle to static receding contact angle. Third stage: contraction of the droplet base when the static receding contact angle remained constant just before the droplet completely vanished. For partial wetting cases contact angles hysteresis is present thus causing the second stage of spreading to exist. For the cases of complete wetting second spreading regime does not exist and is limited to first and third stage only [37,38] as discussed earlier (Fig. 7).

Blood droplet spreading on nitrocellulose membranes demonstrate partial wetting behaviour [38]. For comparison of the spreading behaviour of blood on nitrocellulose membranes, the scaling used in the previous section cannot be used, because spreading kinetics depends now on advancing contact angle. Therefore dimensionless parameters used for partial wetting case were: $\bar{L}=L(t) / L_{a d}, \bar{l}=l(t) / l^{*}$, $\bar{\theta}=\theta(t) / \theta_{a d}$ and $\bar{t}=t / t^{*}$, where $L_{a d}$ is the maximum droplet base radius, $\theta_{a d}$ is the advancing contact angle. In Fig. 14-16 the time evolution of base radius of the blood droplet, wetted radius inside nitrocellulose membrane and dynamic contact of the blood droplet are shown for the partial wetting case. Plots presented in Figs. 14-16 confirm that there exist three stages of spreading, which is a characteristic feature of partial wetting case as discussed earlier (Fig. 7). Fig. 14 clearly shows that the pore size has clear influence on the kinetics of blood droplet's base radius. Red blood cells were able to penetrate in the membrane for higher pore size substrate. Fig. 15 shows that although there is considerable difference between the spreading kinetics of the blood droplet on different pore sized membranes, but wetted radius shows a universal behaviour. Fig. 16 shows dynamic contact angle variation with time on different pore sized membranes. For higher pore size in which more penetration of the red blood cells occurs has a lower dynamic contact angle in comparison to a blood droplet spreading on a smaller pore sized membrane. This behaviour is also seen to be independent of haematocrit levels of blood. However, note that in the case of spreading over $0.2 \mu \mathrm{m}$ pore size nitrocellulose membranes the red blood cells did not penetrate inside the membrane pores and only plasma could penetrate inside. That is, the final radius of the droplet base did not vanish (see Fig. 15) and only stages 1 and 2 are present in this case. This observation opens a completely new possibility to (i) investigate red blood cells and plasma separately and (ii) use this method for non-destructive separation of living cells from aqueous solutions. 


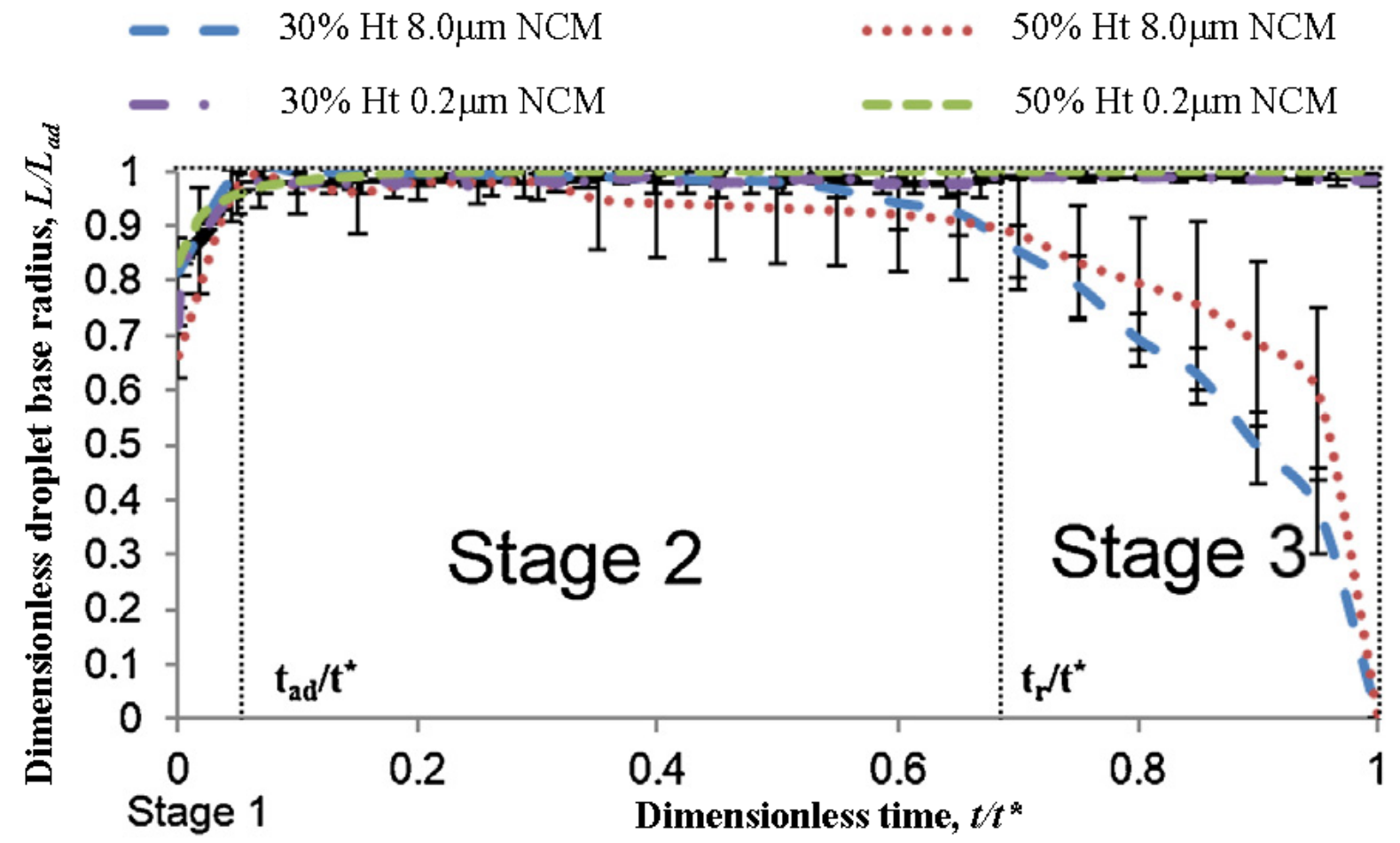

Fig. 14: Comparison between theoretical prediction and experimental results of evolution of dimensionless droplet base radius on dimensionless time for the case of partial wetting of blood samples on $0.2 \mu \mathrm{m}$ and $8 \mu \mathrm{m}$ thickness nitrocellulose membrane (NCM). Reproduced with permission from Ref. [38]. Copyright (C) 2016 Elsevier B.V.

$-30 \%$ Ht $8.0 \mu \mathrm{m}$ NCM

- $\quad 30 \%$ Ht $0.2 \mu \mathrm{m}$ NCM $\cdots \cdots 50 \%$ Ht $8.0 \mu \mathrm{m}$ NCM

$-50 \% \mathrm{Ht} 0.2 \mu \mathrm{m} \mathrm{NCM}$

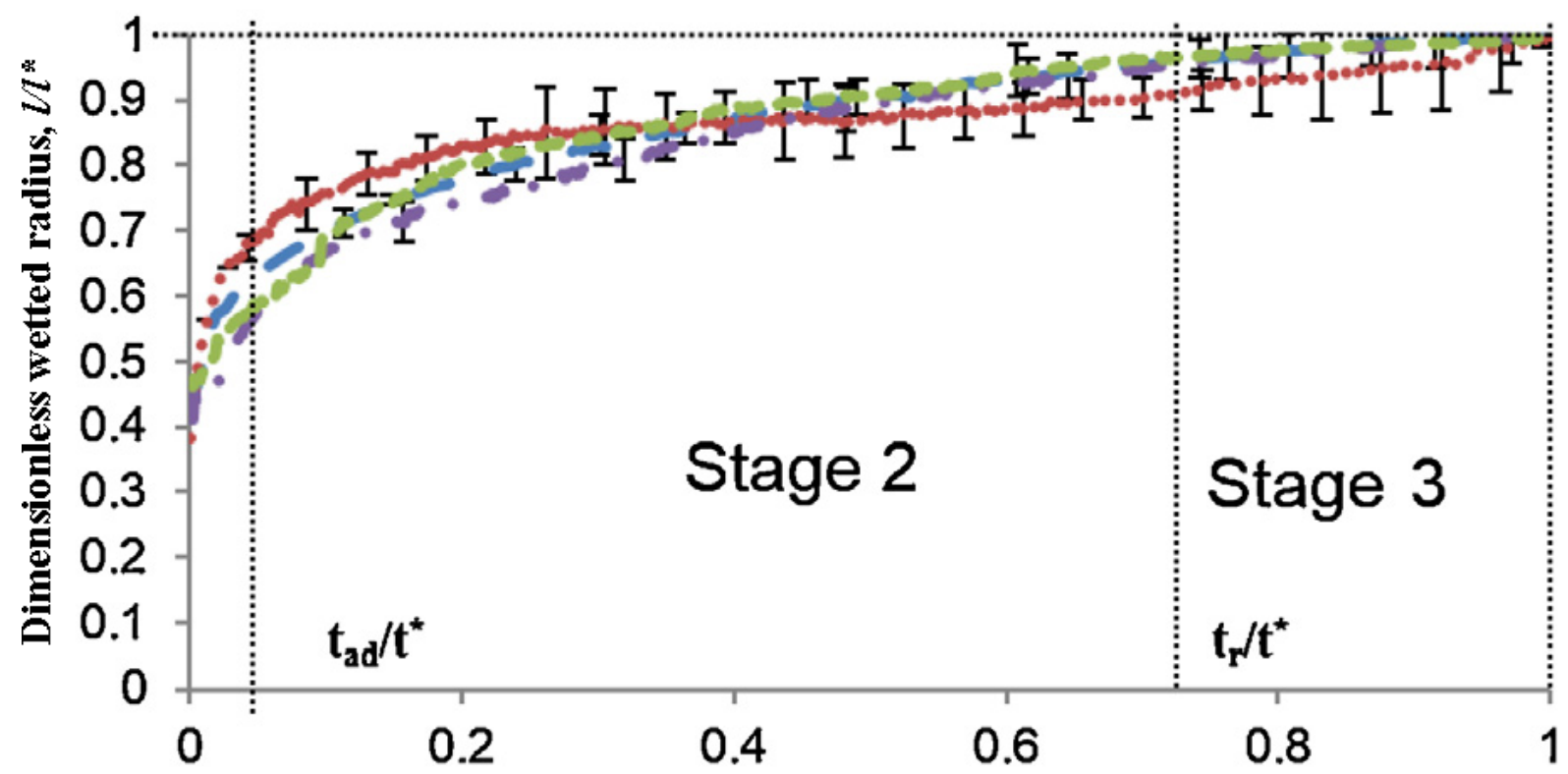

Stage 1

Dimensionless time, $t / t^{*}$

Fig. 15: Comparison between theoretical predictions and experimental results of evolution of dimensionless wetted radius inside porous substrate on dimensionless time for the case of partial 
wetting of blood samples on $0.2 \mu \mathrm{m}$ and $8 \mu \mathrm{m}$ thickness nitrocellulose membrane (NCM). Reproduced with permission from Ref. [38]. Copyright (C 2016 Elsevier B.V.

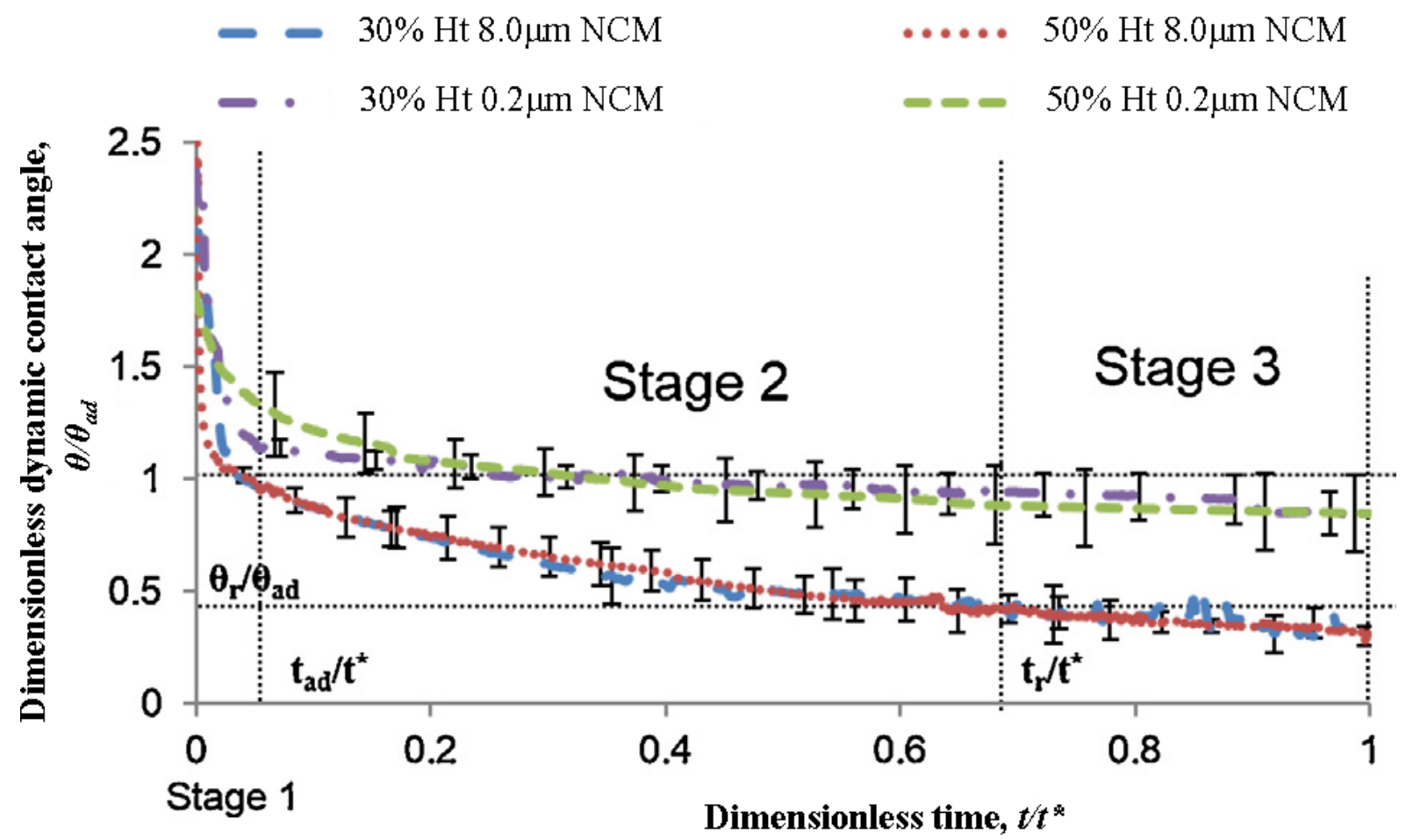

Fig. 16: Comparison between theoretical prediction and experimental results of evolution) of dimensionless dynamic contact angle on dimensionless time for the case of partial wetting of blood samples on $0.2 \mu \mathrm{m}$ and $8 \mu \mathrm{m}$ thickness nitrocellulose membrane (NCM). Reproduced with permission from Ref. [38]. Copyright @ 2016 Elsevier B.V. 


\section{Simultaneous spreading and evaporation kinetics of blood}

Spreading of fluid droplets on rigid surfaces is a universal phenomenon in nature and is presently gaining a lot of interest due to its vast applications in nature and industry. Wetting kinetics of biological fluids has acquired particular appeal, because these are essential to human survival. Blood is a distinct, complex and power law shear thinning non-Newtonian liquid as discussed above [39]. Physical characteristics of blood such as surface tension, density and so on have been reported by Brutin et al. [35]. In order to understand wetting dynamics of blood under diverse conditions, understanding of fundamental of spreading/evaporation kinetics is crucial and is reviewed briefly below.

Initially when a liquid droplet is deposited, the spreading is rapid. This is due to action of capillary and viscous forces [12,16,40]. For Newtonian fluids the spreading exponent of evolution of base radius from Tanner's law is 0.1 . For non-Newtonian fluids deviations from this exponent have been reported [41-43]. Most studies have focused on spreading properties of shear-thinning surfactant/polymer solutions and found that spreading exponent is greater than 0.1, because of the presence of Marangoni stresses due to variation of concentration of surfactants [42].

Brutin et al. [36,44-46] have recently investigated blood droplet wetting, spreading, drying, and evaporation dynamics on solid substrates for biomedical applications. They have been able to identify two regimes of spreading of blood: $L(t) \sim t_{m}$, an early stage where the spreading is influenced by surface tension and viscous forces with a spreading exponent of $m=0.65$ and a later stage determined by the complex colloidal shear-thinning nature of blood with a spreading exponent of approximately $m=0.19$. Both spreading exponents are higher than Tanner's spreading law of $m=$ 0.1 .

Relative humidity, RH, has a significant influence on the spreading/drying process of the blood droplet [36,40]. Zeid and Brutin also suggested that at variable Relative Humidity, RH, conditions the spreading dynamics of blood droplets on glass substrate is quite different. Fig. 17 shows the effect of variation of RH on the spreading of blood droplets before start of the evaporation process. Wetted diameter of the blood droplet is reduced as the RH is increased which is accompanied by the increase in the contact angle that the blood droplet makes with the glass substrate. Blood droplets tend to spread faster at low RH values [40], and the total time to complete the spreading process increases as the RH values increase. Fig. 18 shows that the extent to which the droplet base radius spreads is restricted by RH, i.e. the spread of droplet base radius is less in the case of lower RH. The prime reason for this change is that the relative humidity is directly related to the rate of evaporation, i.e. global rate of evaporation decreases with increase in RH. Therefore spreading/wetting is favoured at higher $\mathrm{RH}$ values. 


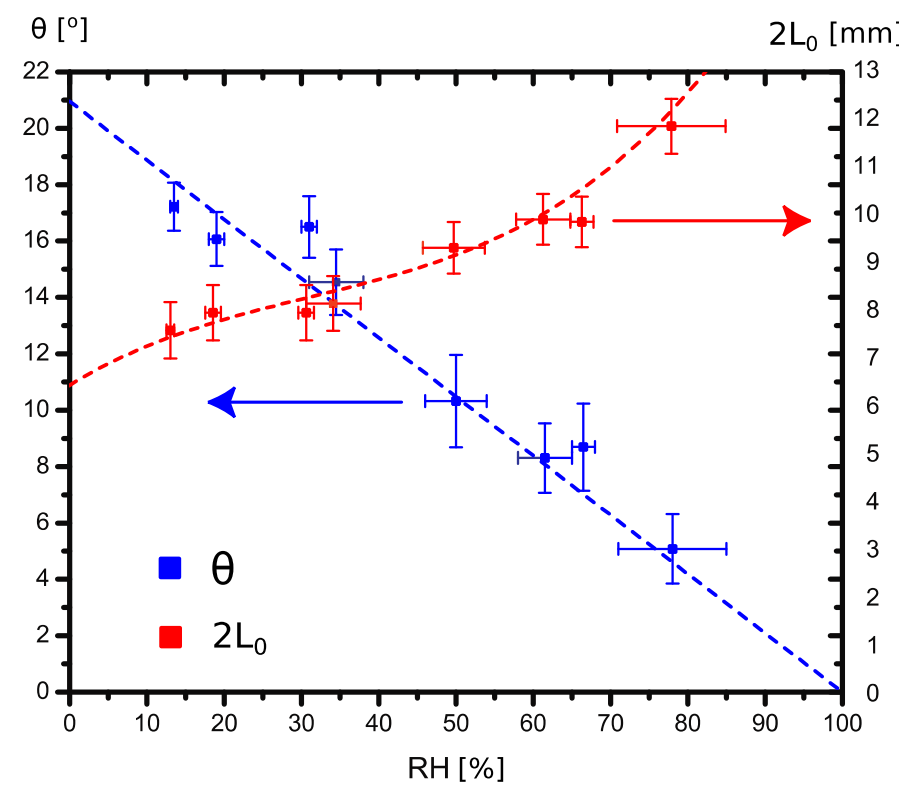

Fig. 17: Effect of variation of relative humidity, $13.5 \% \leq R H \leq 78.0 \%$, on initial blood droplet contact angle, $\theta$, and initial wetted diameter of the droplet, $2 L_{0}$, on a glass substrate. Reproduced with permission from Ref. [36]. Copyright (C) 2013 Elsevier B.V.

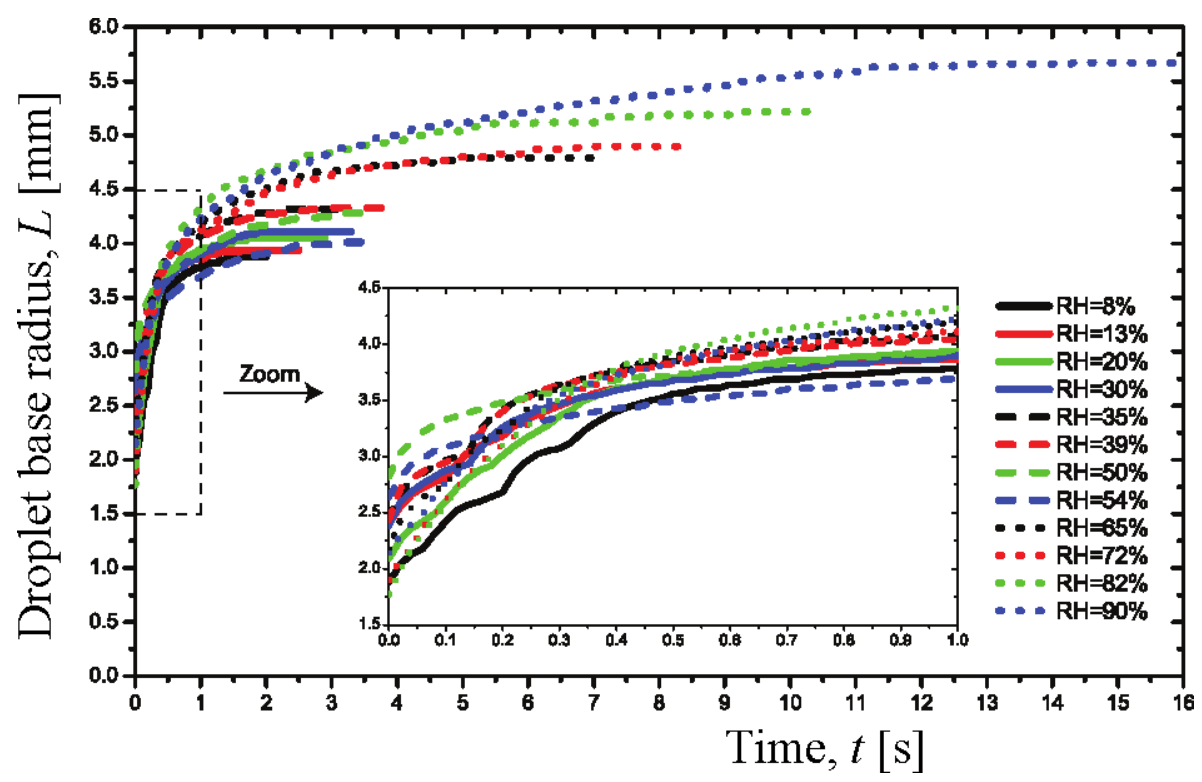

Fig. 18: Effect of RH on the spreading dynamics of the blood droplet base radius. Reproduced with permission from Ref. [40]. Copyright @ 2014 Elsevier B.V. 


\section{Kinetics of evaporation of blood and pattern formation}

In the previous section, the dependency of spreading rate and evaporation rate of the blood droplet on relative humidity was discussed. In this section it is concentrated more on drying by evaporation of the blood droplets and formation of dried patterns. The drying of a droplet of human blood exhibits coupled physical mechanisms, such as Marangoni flow, evaporation and wettability [36,40,45-48]. Complex patterns form during drying/evaporation of suspensions (such as blood) is thoughtprovoking process that occurs in nature as well as industries. Researchers recently have become more interested in highly dried patterns formed by blood, also known as desiccation patterns. Examination of these dehydrated patterns formed by biological fluids have a lot of information stored in them which is used in variety of biomedical applications, such as, DNA micro-array printing [49], disease detection [48,6], and forensics investigations [4].

Dried samples of droplets of blood plasma significantly vary for patients with different diseases [48], see Fig. 19. Analysis of dried patterns formed by blood is used in disease diagnosis. Metastatic carcinoma was first diagnosed by Rapis [50] from dried samples of human plasma. Similarly, Buzoverya and co-workers were able to get pathological data from dried human plasma patterns [51]. Yakhno et al. found the blood serum samples taken from patients suffering from viral Hepatitis B took longer to evaporate than samples from healthy patients [6]. They were also able to identify dried blood plasma from women with premature childbirths had a thick outer crystallized ring.
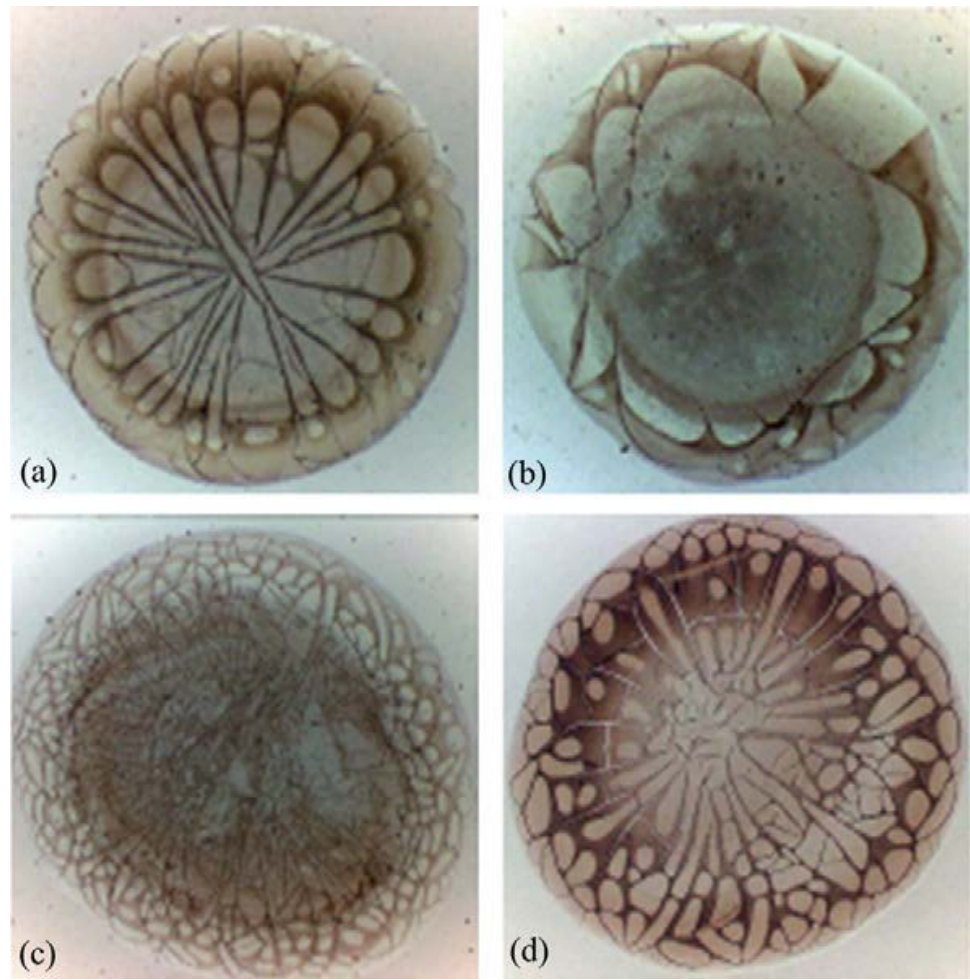

Fig. 19: Dried samples of droplets of blood plasma from (a) healthy patients and from (b), (c), and (d) sick patients. Reproduced with permission from Ref. [48]. Copyright (C) 2010 Elsevier B.V.

In forensics the bloodstains, that were observed, dried due to evaporation and formed crack patterns depending upon health conditions as well as the age group of the victim [49]. Bremmer et al. [52] recently reviewed the physical processes involved in the ageing of bloodstains and the techniques which are used to determine precisely the age of bloodstains. An understanding of the drying process 
helps the investigators in reconstructing the events and to determine the time elapsed after the crime [4].

Brutin et al. have reported different stages of evaporation of blood droplets [35]. Initial diameter of blood droplet used was $5.9 \mathrm{~mm}$. Evaporation time was approximately 36 mins. Blood droplet remains pinned on the glass substrate during the evaporation process. The authors [35] have divided the evaporation process into the following five stages (Fig. 20):

(I) Duration of the initial stage was of approximately 7.2 mins. During this initial stage red blood cells moved from the centre towards the edge of the droplet (contact line), which was receding. Red deposit could be seen to become more prominent over time during this stage. Desiccation had already started to appear at the edge of the droplet which was moving inwards.

(II) During this stage crystallization started to occur at the edge of the blood droplet, which propagated inwards with time. Simultaneously desiccation progressed inwards. Dark red torus was developing due to the accumulation of RBCs near the edge of the droplet. This torus became progressively darker over time. Duration of this stage was approximately 10.8 mins.

(III) It was observed for the next approximately 7.2 mins that the desiccation of dark red torus became more rapid. Droplet centre became lighter red and during this stage desiccation of the whole droplet was nearly complete. Cracks started to appear around the corona which grew inwards with time in the next stages.

(IV) For the next approximately 5.4 mins central part of the blood droplet desiccated at this stage and desiccation of the edge was completed. Composition of central area of the droplet at this stage was mainly white blood cells, little amount of RBCs (see Fig. 22), proteins, and blood platelets.

(V) For the last approximately 5.4 mins larger plaques of the corona were observed, which moved slightly as the desiccation process was concluded. 


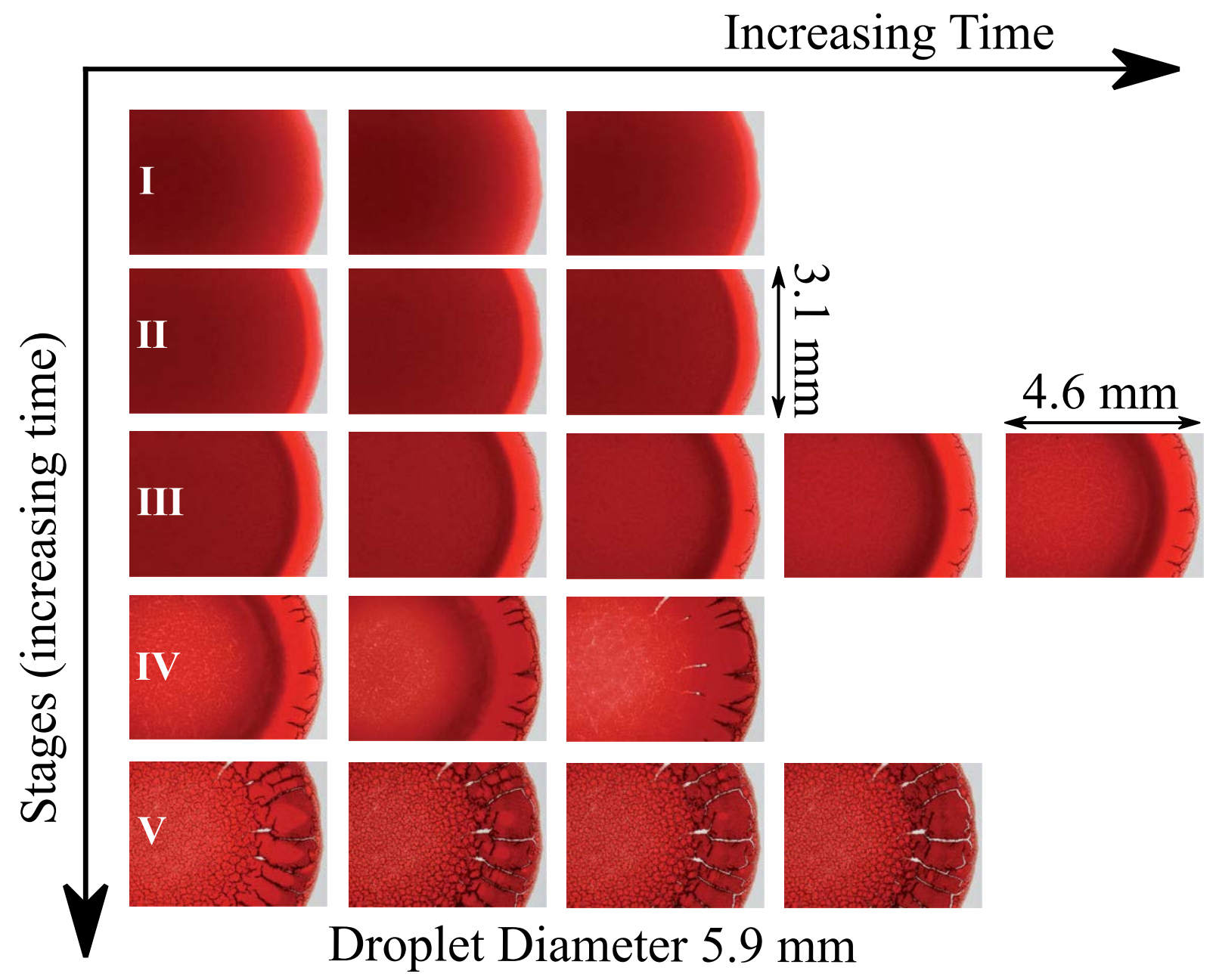

Fig. 20: Stages of drying by evaporation of the blood droplet on a microscope glass slide with time Reproduced with permission from Ref. [35]. Copyright (C) 2011 Cambridge University Press.

Different patterns formed by the totally dried blood droplet on a glass slide can be seen in Fig. 20 . Three main structures/patterns were observed in the dried blood droplet sample, see Fig. 21. In the central region of the droplet, which contains mostly serum, has similar cracks to the ones present at the contact line of the dried blood droplet, small and chaotic plaques were observed. Corona, which is formed by the accumulation of RBCs near the contact line due to Marangoni convection (see Fig. 22), contains larger sized mobile plaques and exists between central region and periphery (well defined and narrow border). Periphery forms the boundary between the initial wetting dried deposit and corona [35], see Fig. 21. 


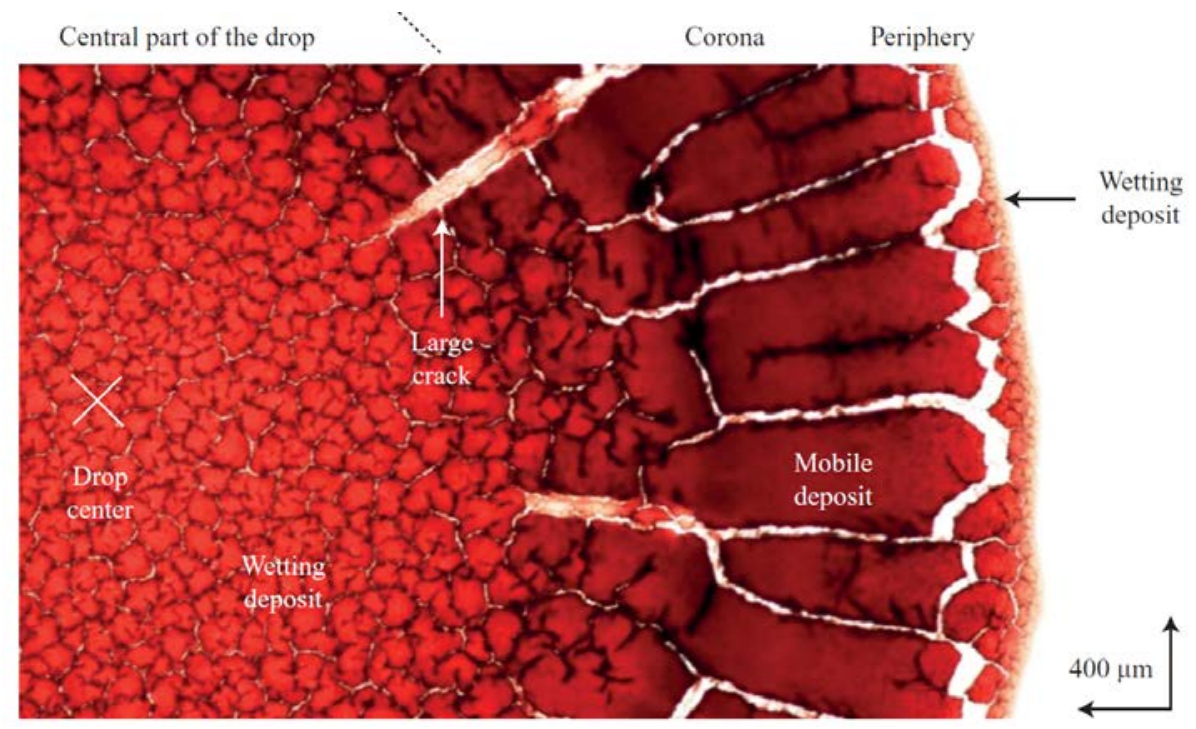

Fig. 21: Zoomed view of different patterns observed in a dried blood droplet. Reproduced with permission from Ref. [35]. Copyright (c) 2011 Cambridge University Press.

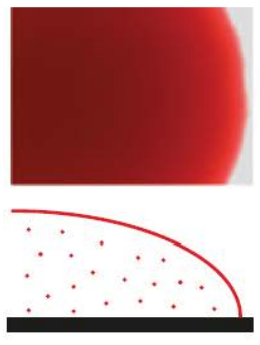

$\mathrm{t}=0$
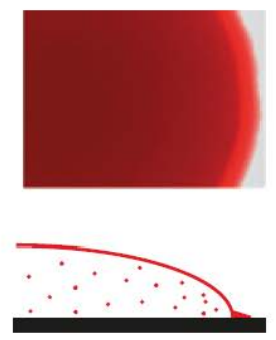

$\mathrm{t} \approx 7.2$
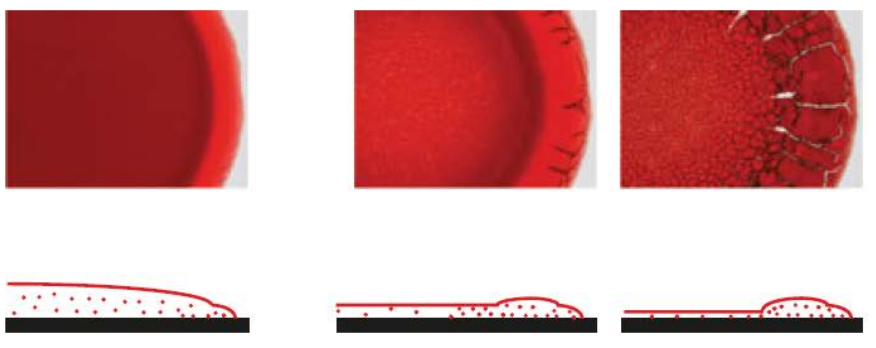

$\mathrm{t} \approx 18$

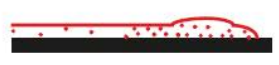

$\mathrm{t} \approx 25.2$

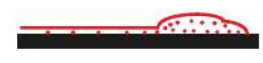

$\mathrm{t} \approx 30.6$

Fig. 22: Movement of RBCs from the centre to the edge of the blood droplet with time (room temperature $29^{\circ} \mathrm{C}$ ). Reproduced with permission from Ref. [35]. Copyright (c) 2011 Cambridge University Press.

Droplet diameter has a prominent influence on the number of cracks developed by the evaporating blood droplet, see Fig. 23. Cracking is observed to take place above a critical diameter of $4.2 \mathrm{~mm}$ where Marangoni convection of RBCs is noticeable. Evaporation of the droplet causes a solid deposit to remain on the glass substrate; this solid deposit accumulates a stress stored inside it during the drying/evaporation process. After this stress is released it causes induction of cracks. Stress inside the dried solid deposit of small droplets is small in comparison to the larger droplets causing less cracks to be observed in smaller droplets.

Substrate wettability also has a considerable influence on the evaporation dynamics of the blood droplet, see Fig. 24 [46]. Brutin et al. used glass $\left(\theta=20.5^{\circ}\right)$, gold $\left(\theta=91.9^{\circ}\right)$ and aluminium $\left(\theta=95.7^{\circ}\right)$ substrates, with different wetting properties for blood spreading/evaporation, which allows observing the drying by evaporation of the blood droplets [46]. Total time of evaporation was influenced by surface wettability, with blood droplet on aluminium to take the longest to evaporate of all the substrates. Evaporative flux took place mainly at the periphery of the droplet for lower contact angle with the substrate (glass) whereas it occurred uniformly around the droplet surface for higher contact angles (gold and aluminium). Uniform evaporative flux on the metal substrates caused gelation and a glassy skin to appear in blood's surface, see Fig. 24. 


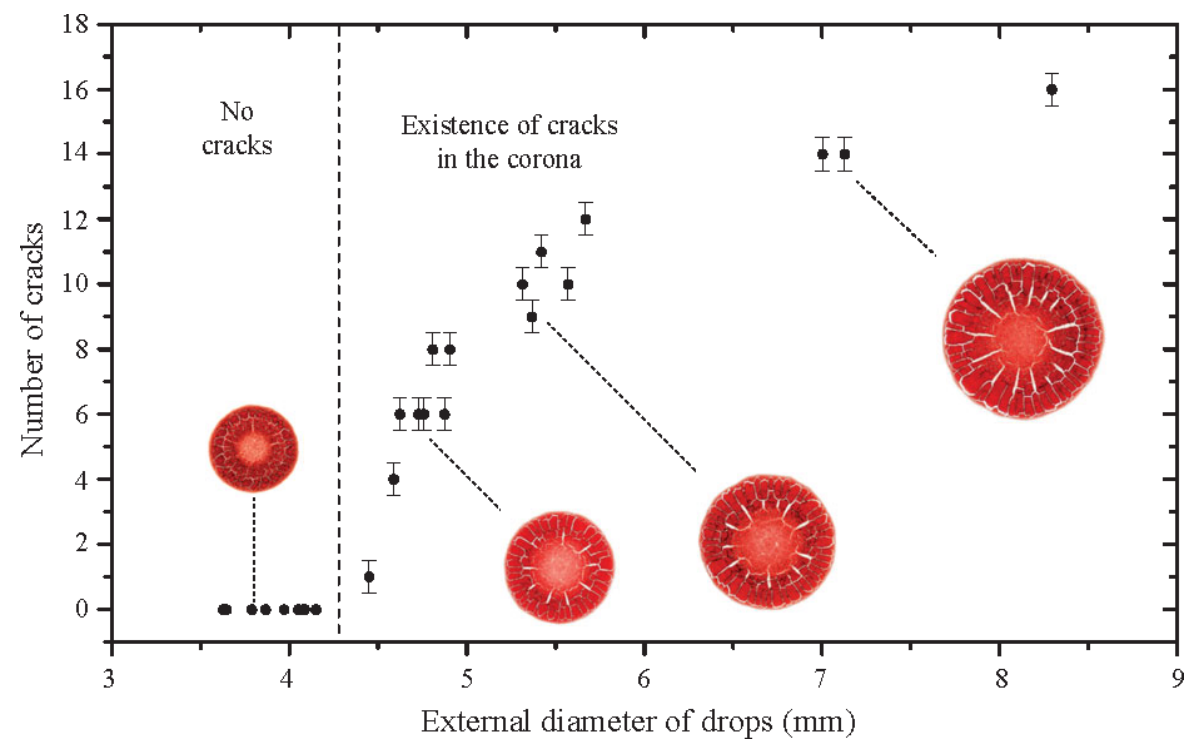

Fig. 23: Number of cracks observed in the corona as the diameter of the blood droplet is varied (room temperature, $22^{\circ} \mathrm{C}$ and relative humidity, $47 \%$ ). Reproduced with permission from Ref. [35]. Copyright (C) 2011 Cambridge University Press.

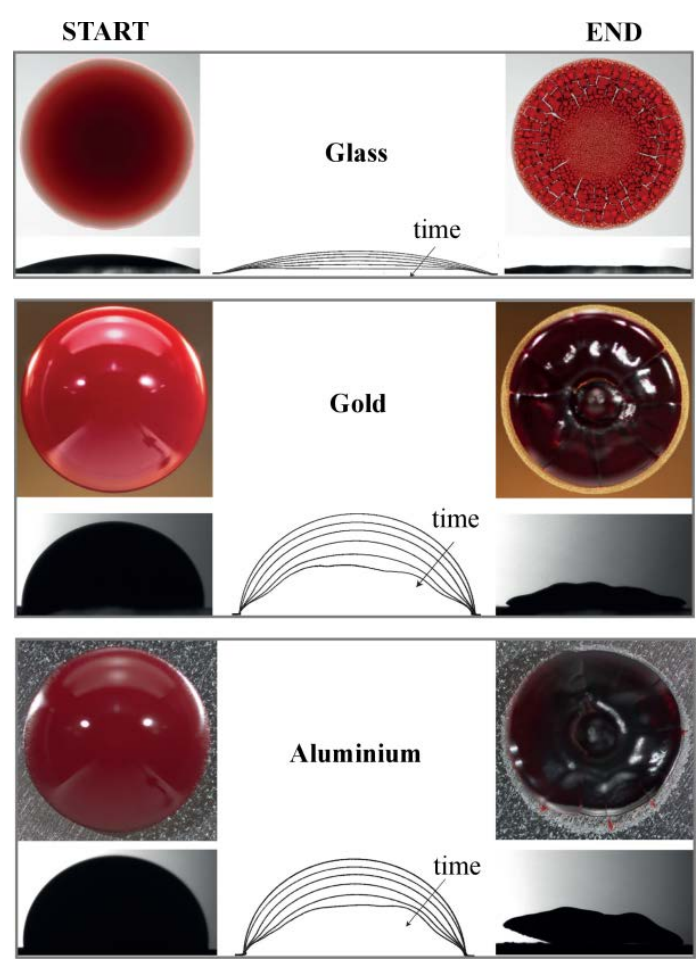

Fig. 24: Initial and final blood droplet shapes before and after completion of evaporation process on Glass, Gold and Aluminium substrate. Reproduced with permission from Ref. [46]. Copyright (C 2012 ASME. 


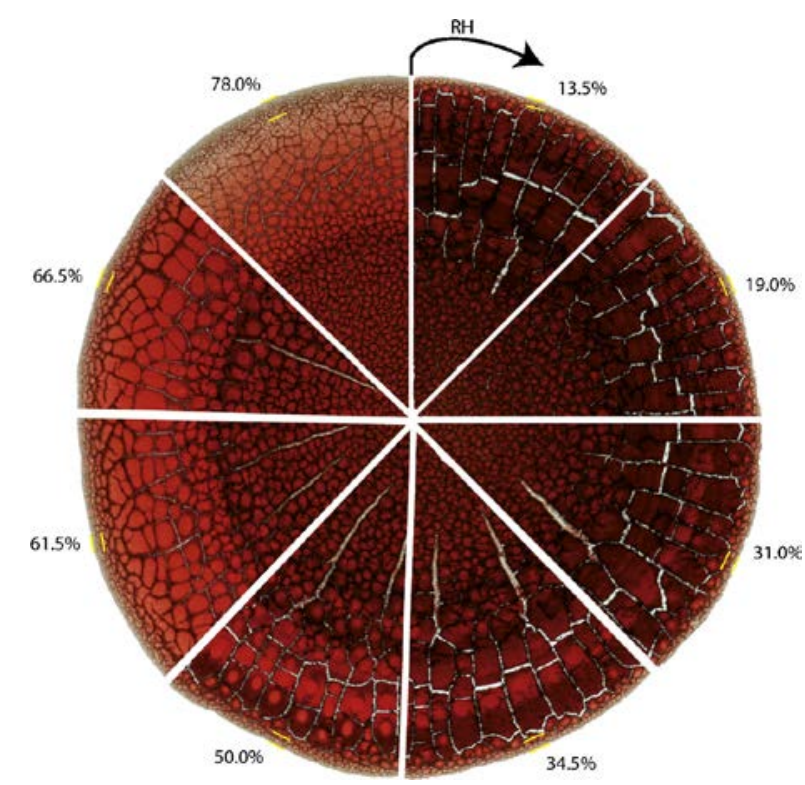

Fig. 25: Final footprints formed by dried blood droplets on glass substrate under the influence of variable relative humidity, $13.5 \% \leq \mathrm{RH} \leq 78.0 \%$. Reproduced with permission from Ref. [36]. Copyright (C) 2013 Elsevier B.V.

Increase in RH clearly depicts changes in the dried blood patterns observed for identical droplet volumes, see Fig. 25. Three different regions are visible as described earlier in Fig. 21. Mobile plaques in the corona are clearly seen to increase in size and the width of the periphery also increases with increasing RH. Drying rate has a direct impact on the mobile plaques sticking to the surface of the substrate. Higher drying rates, i.e. lower RH, the mobile plaques present in the corona of the dried blood droplet are less sticking to glass substrate.

\section{Spreading over hair}

\section{Hair and hair wetting properties}

Hair fibres are constituted by an inner core made from the cortex and medulla (Fig. 26). A protective layer of cuticle cells covers the fibre surface overlapping in a way much like roof tiles. The cells are approximately $0.5 \mu \mathrm{m}$ in thickness individually which tightly overlap to produce a surface layer 6 10 cells thick [53]. A thin hydrophobic covering surrounds each cuticle cell, which is known as the epicuticle made of keratin fibre, and consisting of about 25 wt\% fatty acids, mainly 18methyleicosanoic acid, which is acylated as a thioester [54]. Fig. 26 shows a schematic of a hair fibre. The latter means that hair bunches have a bi-porous structure consisting of pores within each fibre as well as pockets of air between the hair fibres [55]. The cuticle is of great important in cosmetic research since most hair care products including hair shampoos, conditioners and dyes essentially interact with the cuticles [55,56]. For example, shampoos foams play an important role in helping remove dust and extra oil from scalp and hair and these act mostly on cuticles of hair. 


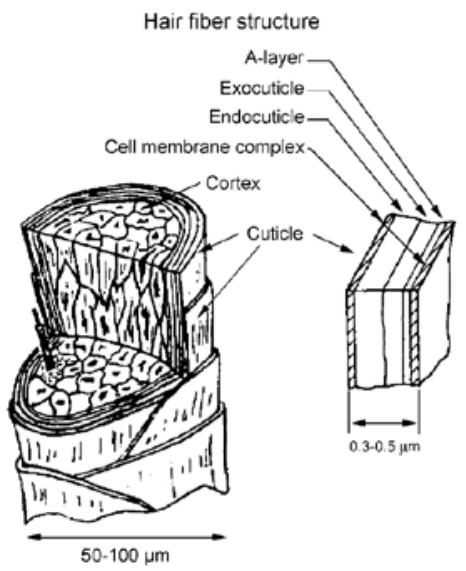

Fig.26: Schematic structure of human hair. Reproduced with permission from Ref. [54]. Copyright (C) 2012 Springer.

Everyone wants hair which looks and feels good, and it is the hair fibre surface which has the ability to change how hair appears on the macroscopic scale. The wettability of hair is of high importance in many cosmetic applications. The spreading of shampoo, conditioner and oils are all carefully engineered for specific purposes which depend on the wettability of the hair. Hair wetting is a phenomenon which is common in day to day life, not only as a result of washing but also occurs when moisture from the air comes into contact with it. When hair fibres are wet, water bridges provide an attractive force, which is responsible for their clumps formation [55,56]. The surface properties of hair fibres are the main parameters which influence wettability of hair. The contact angle is the indicator of the wettability of hair by the product (surfactant, polymer etc.). Complete wetting occurs when the contact angle is $0^{\circ}$, as the droplet turns into a flat puddle [57]. While contact angle is greater than $90^{\circ}$ indicates that wetting of the surface is unfavourable. As a result, the product will minimize its contact area with the surface and form a compact liquid droplet [57]. In other words, with increasing contact angle, the wettability decreases and the wetting area also becomes smaller. Hair care products are aiming to spread well over hair so that they can affect larger contact area.

Hair wettability was studied using various techniques but investigations concentrated on properties of single hair fibre [53-56,58-60]. Lodge and Bhushan used the Wilhelmy balance technique to observe the wetting properties of an individual human hair [58]. The outer lipid layer of a virgin hair is hydrophobic, in contrast to mechanically or chemically damaged hair which is hydrophilic. This is due to the destruction of the lipid layer. The use of conditioner changes the contact angles of both damaged and undamaged hair, raising and lowering it respectively. A large hysteresis of contact angle was observed for virgin hair, however, the treated hair had smaller hysteresis values. It was also shown, that the type of damage, chemical or mechanical, has a significant effect on the results; chemically damaged hair exhibits a more hydrophilic nature than mechanically damaged hair [58]. The electrostatic properties and the wetting behaviour of the human hair surface at the nano-meter scale have been investigated by using atomic force microscopy by Dupres et al., [56]. Wetting experiments were used to map the local surface changes of hair following the removal of lipids. The combination of wetting experiments and AFM was proposed for mapping finely the local surface changes of hair upon lipids removal. In [59] authors investigated how surface deposits affect the wetting force curve on a single hair fibre treated with polymer JR-400. In [60] the effect of temperature on the deposition of polymers and surfactants on hair fibres was investigated. It was observed that the adsorption of cationic surfactants with low molecular weights increases with temperature, and reaches a maximum at about $60^{\circ} \mathrm{C}$ due to the repulsion of adsorbed species. It was 
also found that an increase in temperature leads to an increase in site activation and improved surface coverage. These results are confirmed by Lodge and Bhushan [58].

Until now little attention has been payed to properties of hair bunch although these properties are not identical with a single fibre of hair and crucial regarding applications including spreading of shampoo and hair care products. Single hair could not represent comprehensive hair behaviours especially on measuring contact angle and wettability. Single hair fibre should be considered as the porous structure itself but a bunch of hair has a bi-porous structure consisting of pores within each fibre as well as pockets of air between the hair fibres. The wettability on hair tress was investigated for the first time in [61].

\section{Hair products, Aculyns polymeric solutions}

Nowadays hair care products like shampoo which only clean hair do not satisfy consumers' requirements; therefore various ingredients (including surfactants, polymers, additives) are incorporated into the product to meet demands of consumers [54,62].

Cationic surfactants were generally recognized as conditioners or sunscreen to absorb UV light or vehicles for other ingredients [63]. Anionic surfactants are of high foaming ability and skin affinity so that are widely used as ingredients of shampoo. In conventional hair care products, foams are stabilised by surfactants, but recently polymers replace the role of surfactants $[64,65]$. Polymers have a similar effect as low-molecular surfactants which adsorbed on the interfaces and stabilize foam films [65]. Two polymers used widely in the cosmetic field are polymer emulsions Aculyn ${ }^{\mathrm{TM}} 22$ and Aculyn $^{\mathrm{TM}}$ 33. They have a well-established toxicological profile, therefore, they can be used in hair care products [66-68]. It is proved that they are compatible with many non-ionic and anionic surfactants and show synergistic interaction with these surfactants $[67,68]$. Aculyns are used in shampoos, cleaners, shower gels, skin masks, moisturizing creams, lotions and hair colorants. Aculyn $^{\mathrm{TM}} 22$ (A22) is known as a hydrophobically-modified alkali soluble emulsion (HASE) (Fig. 27) [67] and Aculyn $^{\mathrm{TM}} 33$ (A33) is an anionic alkali soluble polymer emulsion which is lightly crosslinked [68] (Fig. 27).
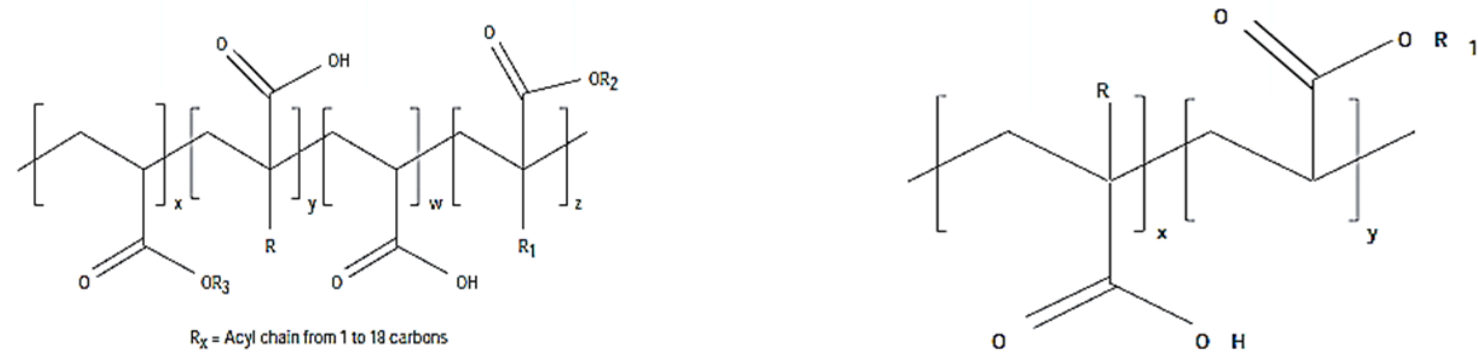

Fig. 27: Schematic structure of Aculyn ${ }^{\mathrm{TM}} 22$ (left) and Aculyn $^{\mathrm{TM}} 33$ (right). Reproduced with permission from Refs. [67,68]. Copyright (c) 2006 Rohm and Haas.

\section{Rheology of Aculyns solutions}

Rheology of Aculyns solutions was studied in [64,65]. As shown in Fig. 28 Aculyn $^{\mathrm{TM}} 22$ and Aculyn $^{\mathrm{TM}} 33$ solutions exhibit non-Newtonian shear-thinning power law behaviour, $\mu=K \dot{\gamma}^{n-1}$. The 
rheology of these two polymers is quite similar: both are shear thinning non-Newtonian power law liquids and their solubility in water decrease with $\mathrm{pH}$.

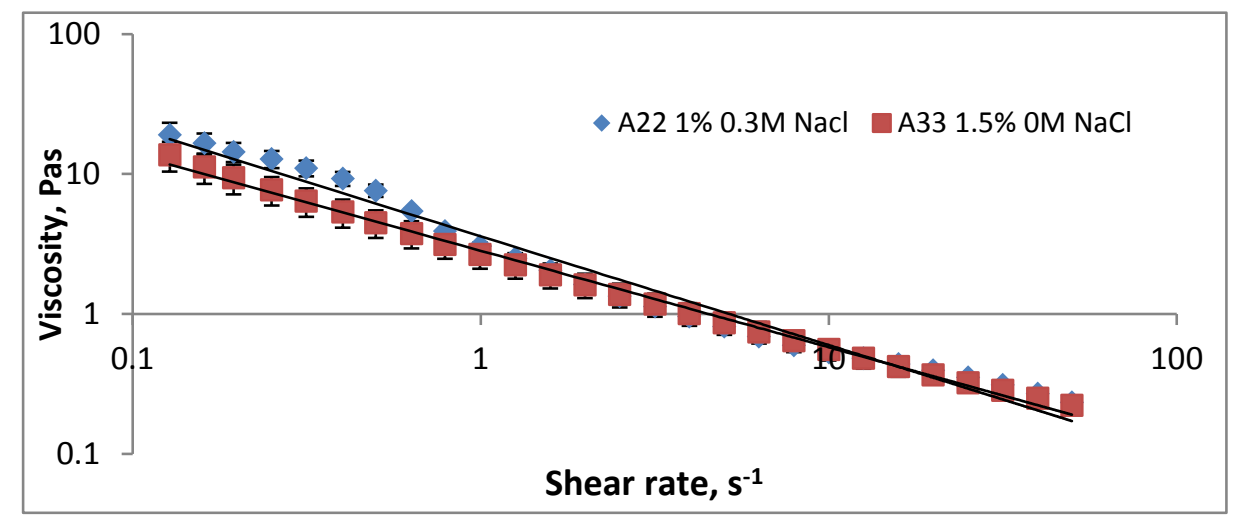

Fig. 28: Dependency of the viscosity of the A22 and A33 solutions on shear rate. Fitted according to $\mu=K \dot{\gamma}^{n-1}$

It was observed in [64] that an increase in salt concentration cause the viscosity of both solutions to decrease in a similar fashion, as seen in Fig. 29. Fig. 30 shows that solution viscosity increases with the increase of the polymer concentration [65]. The viscosity of the A22 solutions is much higher than that of the A33 solutions at the same polymer concentration.

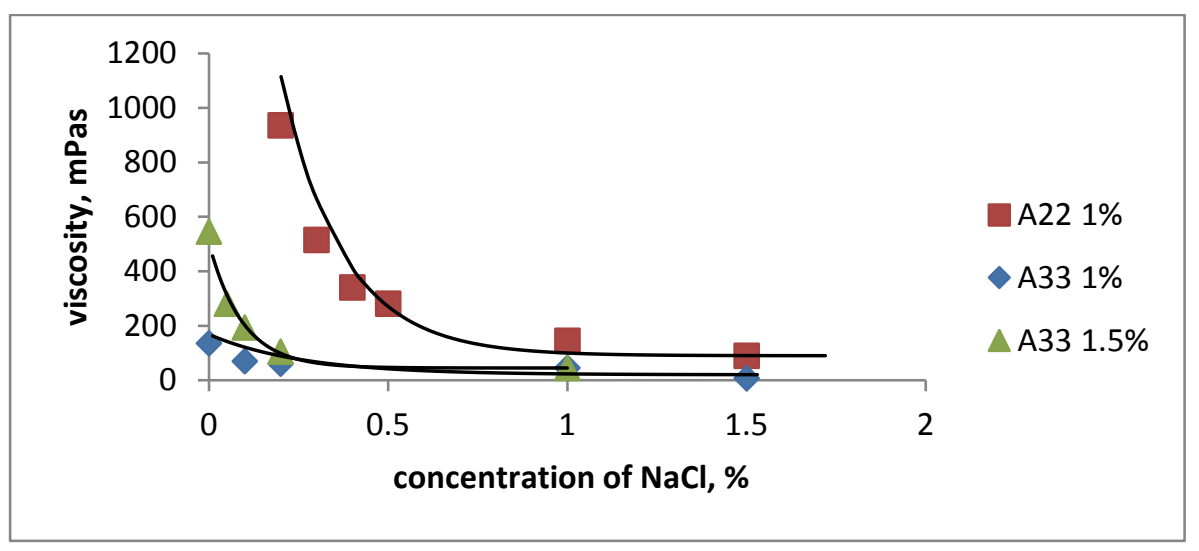

Fig. 29: Viscosity of A33 $1 \%$ (1), A33 $1.5 \%$ (2) and A22 (3) $1 \%$ solutions at shear rate of $12 \mathrm{~s}^{-1} \mathrm{vs}$ concentration of $\mathrm{NaCl}$ salt. 


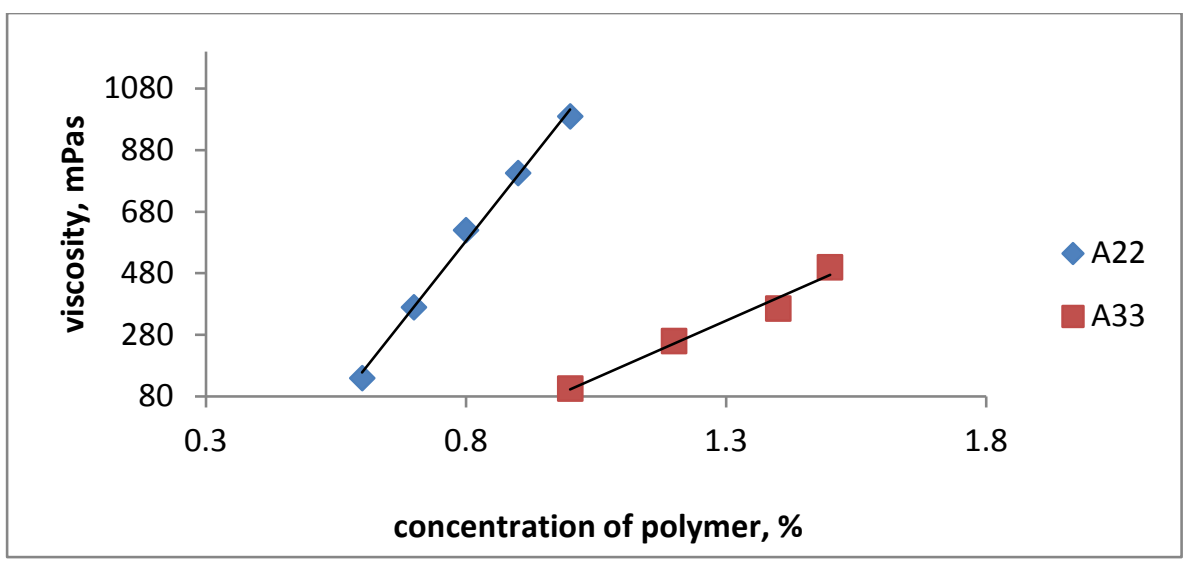

Fig. 30: Dependence of the viscosity of A22 and A33 solution on the concentration of the polymer.

A22 solutions were found to demonstrate yield stress in contrast to A33 solutions which show zeroyield stress in most samples [64]. The effect of additives on the rheological properties of the A22 and A33 solutions was studied in [65]. It was found that addition of iso-propanol results in a decrease of the bulk viscosity of A22 solution whereas the viscosity of A33 remains unchanged. For A22 solutions containing iso-propanol the region of pronouncing shear thinning behaviour is moved to the smaller shear rates.

\section{Spreading of polymeric solutions over hair tress and wetting transition}

It is important to notice that behaviour of hair care products is different when they are applied on wet or dry hair; generally, wetting should be studied in the conditions which can mimic real applications. The majority of research presented in the literature concentrate on the interaction of a single hair fibre (dry or wet) with various liquids [56,58-60]. As it was mentioned earlier the wetting properties of the bunch of hair were investigated for the first time in [61]. Wetting of dry hair tresses by polymer solutions is largely unexplored up to now. However, these properties attract significant interest from the industry, particularly for treatments of dry hair, e.g. for hair colouring products, hair styling products, leave-on conditioners, and serums. There is a requirement to minimise the use of harsh surfactant in such systems; cosmetic polymers with a pronounced affinity to hair interfaces, like Aculyn $^{\mathrm{TM}} 22$ and Aculyn ${ }^{\mathrm{TM}} 33$ solutions, is one of such alternatives. In the research presented in [61] bunch of hair was used to determine the behaviour of polymeric solutions during real cosmetics applications. Aculyn ${ }^{\mathrm{TM}} 22$ and Aculyn ${ }^{\mathrm{TM}} 33$ polymer emulsions may contain some trace amounts of surfactant (sodium dodecyl sulphate), therefore some low level of surfactant was expected in the experiments [61]. Effect of the surfactant and other additives on polymer solutions rheology and wetting properties was separately investigated in $[61,65]$. In the referred works $[61,64,65]$ polymers were used without any pre-processing, like purification to keep the condition of the research experiments as close to the real application conditions as possible. Wetting properties of the polymeric solutions on the hair tresses by contact angle measurements were investigated in [61]. Blended human hair tresses (brown, Caucasian origin) were used and arranged into flat tresses of 12 $\mathrm{cm}$ length, width $2.5 \mathrm{~cm}$ and approximately $3 \mathrm{~mm}$ thickness. The average weight of the hair tress was $2 \mathrm{~g}$ and the average number of hair fibres in the tress was around 4000. For wetting measurements the tresses were secured in a special custom made frame to provide as much hair alignment as possible. Frame allows fixing a bunch of hair in a way which enables measurement of contact angle on hair. The surface of hair tress was made as flat as possible. An example of hair arrangement is presented in Fig. 31. 


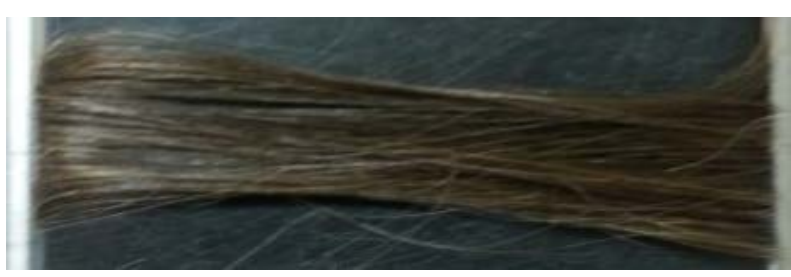

Fig. 31: Example of hair tress - support for contact angle measurements.

The thickness of the tress was sufficient to avoid contact of the investigated liquid with the frame material. There were some expected variations in the arrangement of individual hair fibres on the frame in the repeat experiments, leading to the increased standard error of measurements. The porosity of the tress and distance between fibres were estimated [61]. The volume of the hair tress was around $9 \mathrm{~cm}^{3}$ (an approximation of the cuboid with dimensions $12 \mathrm{~cm} x 2.5 \mathrm{cmx} 0.3 \mathrm{~cm}$ ), the average volume of liquid absorbed by hair tress was $3.6 \mathrm{ml}$.

The porosity of hair tresses was estimated using equation (18):

$$
\varepsilon=\frac{V_{l}}{V_{t o t}}
$$

where $\varepsilon$ is the porosity, $V_{l}$ is the volume of liquid that can be accumulated within the bunch of hair and $V_{t o t}$ is the total volume of hair tresses. Using experimental measurements and Eq. (18), the porosity of bunch of hair was estimated as $\sim 0.43$.

In addition, the distance between the fibres can be estimated using the following equation:

$$
\varepsilon=1-\frac{\pi R_{f}^{2}}{\left(D+2 R_{f}\right)^{2}},
$$

where $D$ is the distance between the fibres and the radius of fibres, $R_{f}$, is about $50 \mu \mathrm{m}$. According to Eq. (19) the distance between the fibres was estimated as $\sim 17.4 \mu \mathrm{m}$.

The behavior of the polymer solutions on hair tresses is complex, because multiple processes such as spreading, evaporation, and penetration into the hair tress can take place simultaneously. It is not possible in these experiments to determine directly which phenomenon dominates. In the research presented in [61], behavior of liquids on hair over relatively long time frame (up to 30 minutes) was observed; distinction between the spreading time (which can be directly identified) and the process time, which is the time between depositing a droplet on a hair tress, and the complete disappearance of the droplet, was made.

A hair tress demonstrates hydrophobic properties not only because of the hydrophobic nature of the hair surface but also because of air pockets in-between the fibres. That is, three wetting regimes: (a) Cassie wetting, where liquid is sitting on the hair tress, without penetrating inside the tress, (b) Wenzel wetting, characterized by penetration of liquid into the hair tress, (c) transition from Cassie wetting to Wenzel wetting, after some critical contact angle is reached were observed in [61].

The solutions of Aculyn ${ }^{\mathrm{TM}} 22$ and Aculyn ${ }^{\mathrm{TM}} 33$ polymers spread on hair tresses. However, show markedly different behaviour which is presented in Figs. 32 and 33. 


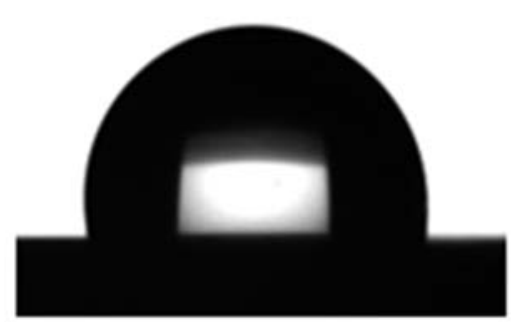

$\mathrm{t}=0$ (second)

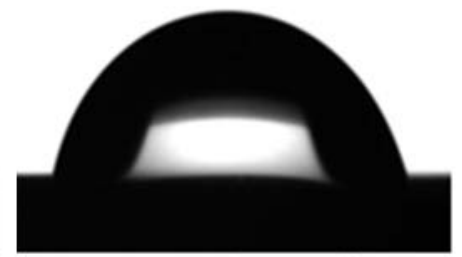

$\mathrm{t}=20$ (second)

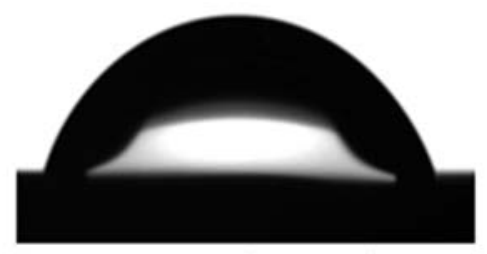

$\mathrm{t}=197$ (second)

Fig. 32: Behaviour of pure A22 solutions on a hair tress.

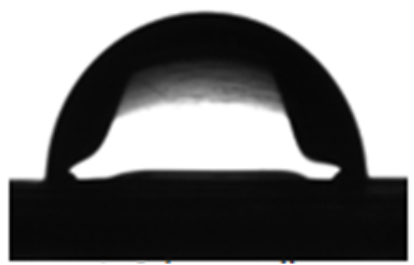

$\mathrm{t}=0$ (second)

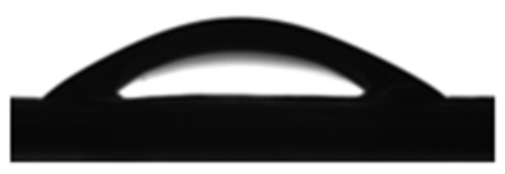

$\mathrm{t}=40$ (second)

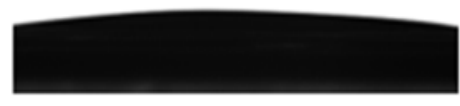

$t=200$ (second)

Fig. 33: Behaviour of pure A33 solutions on a hair tress.

For the A22 solutions, the droplet remains on the surface of the hair for almost half an hour (Fig. 31), and only slow (if any) imbibition was observed. For the A33 solutions, the complete penetration/imbibition happens fast; after the contact angle reaches a critical value (around $60^{\circ}$ ) droplet penetrate into bunch of hair, see Fig. 33 [61].

Dynamic contact angle and base line diameter (DB) of the A22 polymer solution droplets on the hair tress is presented in the Fig. 34. The initial contact angle on the hair tress for this polymer was about $100^{\circ}$. Spreading stopped after $10-20 \mathrm{~s}$, and the contact angle had reached the value of $80^{\circ}$ (Fig. 34). The droplets remained on the surface of the hair tress after the initial fast spreading stage. Following this stage, the total process time (until the droplet disappearance) was around $2000 \mathrm{~s}$. During this later stage, slow evaporation and possible imbibition of the solutions into the hair were observed. Interestingly, wetting properties of the investigated solutions were very similar regardless of large differences in viscosity [61].
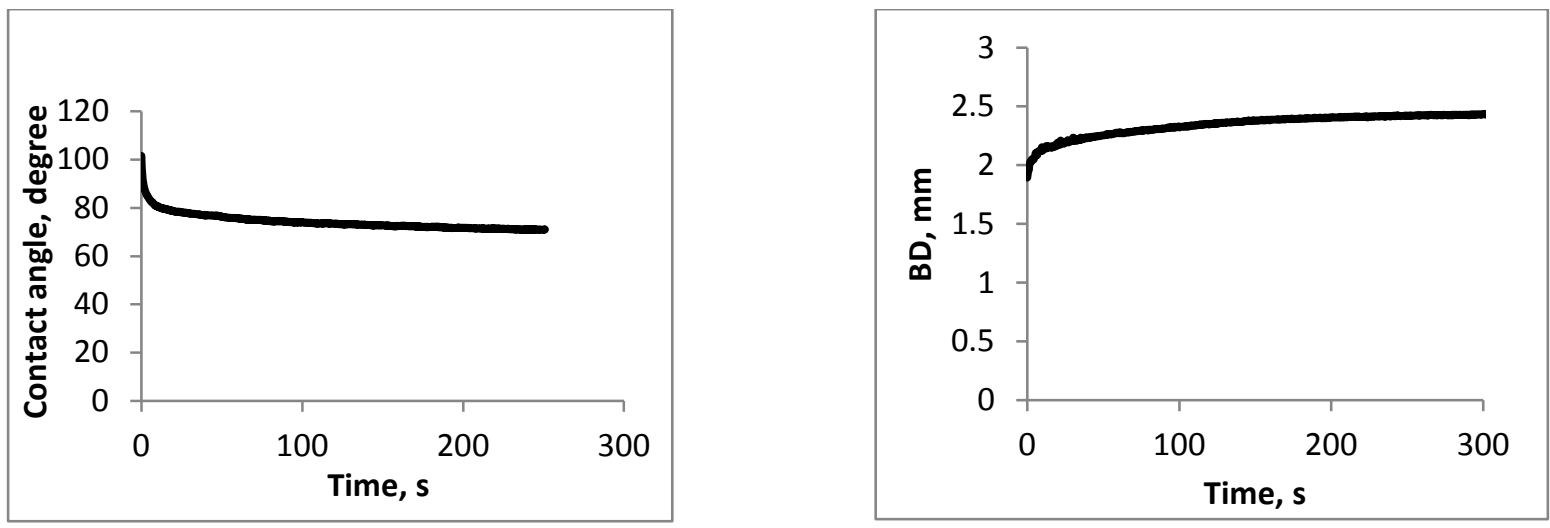

Fig. 34: Contact angle and base line diameter of A22 1\% 1.3M NaCl solution on a hair tress. 
Characteristic droplet shape of the A22 solution on the hair tress is presented in the Fig. 32. Droplet remained on top of the tress after initial short time spreading. Droplet volume was decreasing with time due to evaporation and, possibly, slow penetration inside the hair tress.

The initial contact angle of the A33 solutions on the hair tress was about $100^{\circ}$. For the A33 $1 \% 0 \mathrm{M}$ $\mathrm{NaCl}$ the first fast stage of spreading was completed after 5-25 s. For the $\mathrm{A} 331.5 \% 0 \mathrm{M} \mathrm{NaCl}$, this stage took 10-30 s. In both cases the final contact angle after spreading was around $60^{\circ}$. The total process time, during which the droplet disappeared completely, was $100 \mathrm{~s}$ for $\mathrm{A} 331.5 \% 0 \mathrm{M} \mathrm{NaCl}$ (Fig. 35) and $200-250 \mathrm{~s}$ for $\mathrm{A} 331 \% 0 \mathrm{M} \mathrm{NaCl}$ (Fig. 36). It is important to notice that the droplet with higher viscosity disappeared first [61].
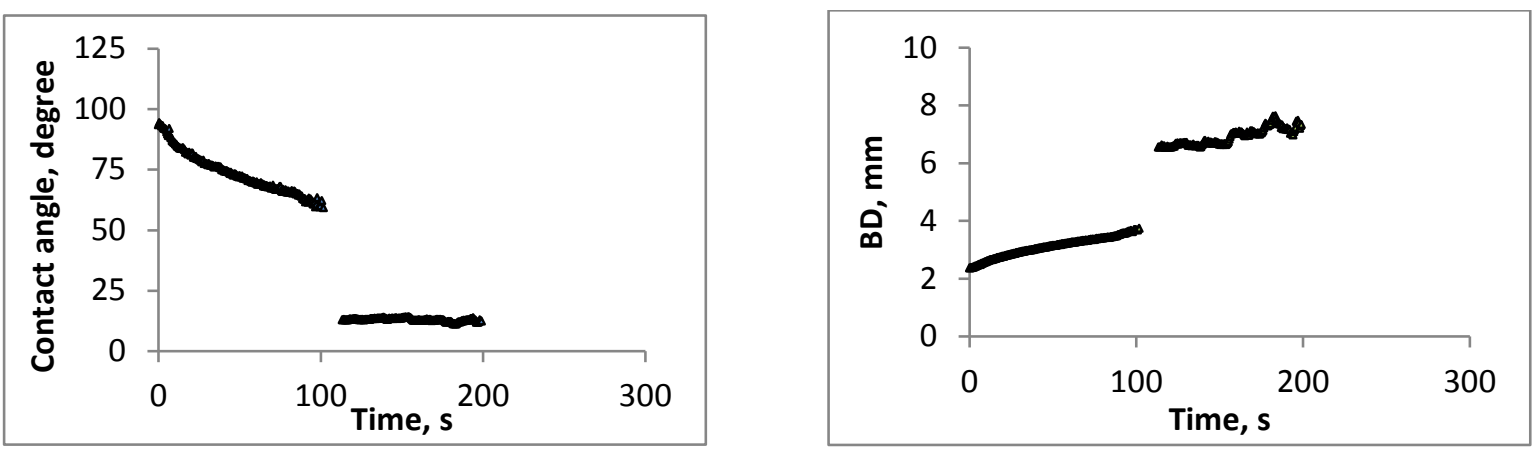

Fig. 35: Contact angle and base line diameter of A33 1.5\% $0 \mathrm{M} \mathrm{NaCl}$ solution on a hair tress.
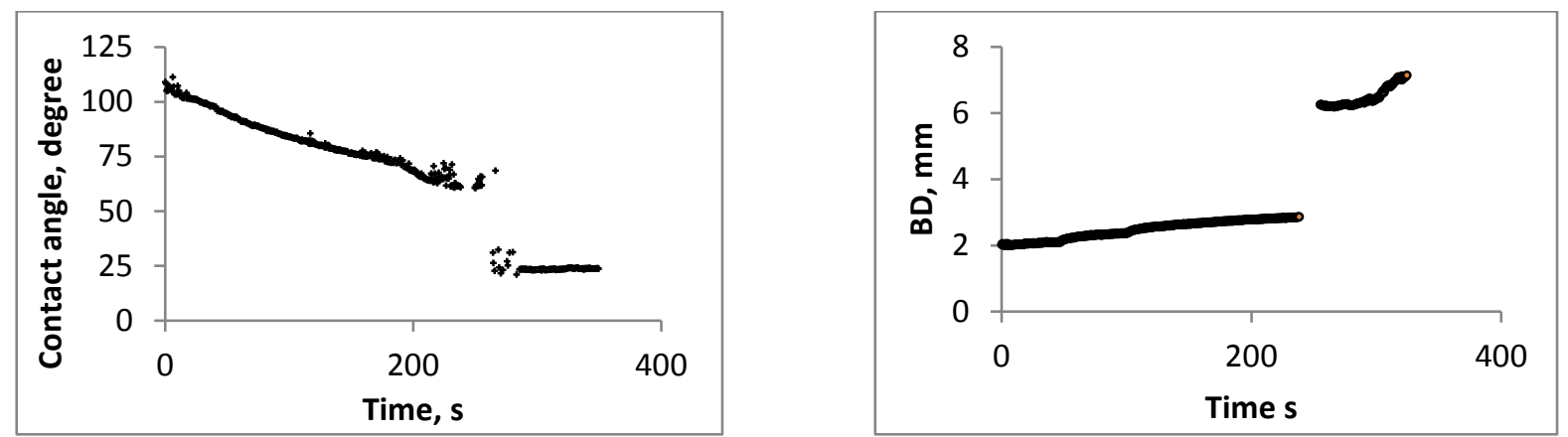

Fig. 36: Contact angle and base line diameter of $\mathrm{A} 331 \% 0 \mathrm{M} \mathrm{NaCl}$ solution on a hair tress. Reproduced with permission from Ref. [61]. Copyright @ 2015 Elsevier B.V.

Figs. 35 and 36 shows that in the case of A33 solutions, where the contact angle reached the critical value (around $60^{\circ}$ ), fast penetration into the hair tress was observed. This resulted in a "jump" on the graph of contact angle and base line diameter, as seen on the Figs. 35 and 36. This can be explained by a so-called Cassie-Wenzel wetting transition which is caused by filling the pores inside the porous media (like the hair tress in the case) by liquid [69-72]. Schematic penetration of the liquid inside the porous media (hair tress) is presented in the Fig. 37. In the case of Cassie stage, liquid wetted only the first layer of fibres; however, at the Wenzel stage liquid penetrated inside the hair tress. After the transition, capillary transport along fibres becomes also possible [61]. 


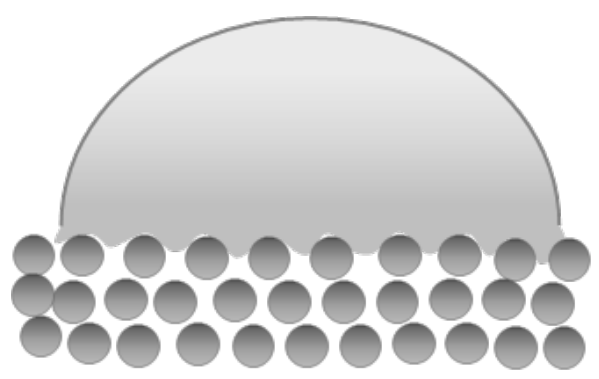

Cassie stage

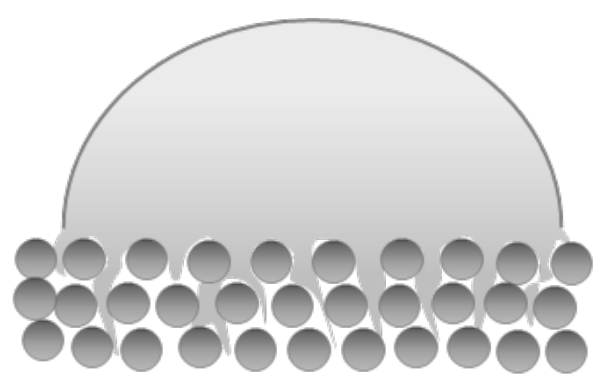

Wenzel stage

Fig. 37: Behaviour of a liquid droplet on the hair tress. Cassie stage - only first layer of fibres is wetted; Wenzel stage - liquid penetrate dipper into the hair. Reproduced with permission from Ref. [61]. Copyright (c) 2015 Elsevier B.V.

It was found in [61] that the condition for wetting transition is to reach the critical contact angle, which appears to be around $60^{\circ}$. This condition is satisfied for the A33 solutions and not satisfied for the A22 solution, the latter showing higher contact angle. The critical value of contact angle was obtained faster for the A33 1.5\% solution than that for the A33 1.0\% solution. It can be caused by the slightly higher concentration of the trace surfactant carried over from the stock emulsion with the higher concentration of the polymer. The decrease of the total process time for the A33 solutions with added SDS confirms this observation. Differences in the wetting behaviour of investigated solutions can be explained in terms of differences in polymer chemistry. A22 is a hydrophobically-associative polymer and has strong intermolecular associations under certain conditions and A33 is a microgeltype thickener that is not capable of strong intermolecular associations. It could be a reason why A33 solutions can reach critical contact angle on hair and penetrate into hair tress.

Influence of additives on wetting properties of polymeric solution over hair tress was also investigated in [61]. It was found that addition of a surfactant or a solvent (SDS and i-propanol) can improve wetting properties of both polymers. In the case of the relatively low viscosity A22 solution (1\% polymer, $1.3 \mathrm{M} \mathrm{NaCl}, 100 \mathrm{mPa} . \mathrm{a})$ addition of $10 \%$ i-propanol causes immediate imbibition of the solution into the hair. For a mixture of A22 and A33, the wetting properties follow the behaviour of the polymer with the higher volume fraction.

\section{Foam drainage}

Polymer-surfactant mixed solutions are frequently used to form foams for cosmetics applications [73]. A mixture of anionic surfactant with anionic polymers (mentioned earlier Aculyns) has been used to investigate the foaming properties of polymer-surfactant mixed solutions and drainage kinetics of foams produced from those solutions [64,65]. It was found in [64] than both polymers have a high foamability and form stable foam. Drainage kinetics is determined by the bulk viscosity rather than the surface viscoelasticity, what is in line with theoretical estimations for the measured values of these parameters. However, for the highly viscous (high shear rate viscosity about $500 \mathrm{mPa} \cdot \mathrm{s}$ ) A33 solution the drainage after 20 min slows down in comparison to the A22 solution of the similar viscosity. Influence of additive on foaming properties of the polymeric solutions and foam drainage was investigated in [65]. It was found that concentrations of polymers, sodium chloride and iso-propanol alcohol are useful parameters to control properties of A22 and A33 solutions and, hence, kinetics of foam drainage and foam stability.

As a bulk viscosity of foaming solution is a crucial parameter for foam drainage it is important to notice that the bulk viscosity of A22 solutions is much higher than that of A33 solutions at the same composition. The viscosity of mixture solution increases with the increase of the A22 content. The 
addition of iso-propanol results in a decrease of the bulk viscosity of A22 solution whereas the viscosity of A33 remains unchanged. It is important to take into account that polymeric solutions used in $[64,65]$ are non-Newtonian shear thinning liquids for which the changes of viscosity with the shear rate can depend strongly on the composition. For A22 solutions containing iso-propanol the region of pronouncing shear thinning behaviour is moved to the smaller shear rates. The last effect depends on the salt concentration. The bulk viscoelastic modulus, $\mathrm{G}(\mathrm{Pa})$, and the surface viscoelastic modulus, $\mathrm{E}$ $(\mathrm{mN} / \mathrm{m})$, increase with the increase of polymer concentration in solutions and with the decrease of the salt concentration [65]. The addition of iso-propanol lowers the surface viscoelasticity of both polymeric solutions, but the effect is much more pronouncing for those of A22. The drainage kinetics of foams prepared using solutions with the addition of $10 \% \mathrm{i}-\mathrm{prOH}$ is faster than that of foams without iso-propanol, especially for A22 solutions [65]. The considerable decrease in the surface viscoelastic modulus due to the addition of iso-propanol results in a faster foam coarsening. The considerable decrease in the foam stability was noticed for the sample with the lowest value of the surface viscoelastic modulus.

A mathematical model of free drainage of foam built up by a power-law non-Newtonian liquid was developed by Starov et al. [74]. A model for free drainage of foams produced from power-law nonNewtonian shear thinning liquids was presented and compared with experimental data for the drainage of foams formed by A22 and A33 polymeric solutions in [74]. The equation was deduced, which describes the drainage of foam build up by a power-law non-Newtonian liquid. The boundary condition at foam/liquid interface was found as a constant liquid volume fraction corresponding to the maxi-mum liquid volume fraction inside the foam.

\section{Foam drainage on porous materials}

In a number of applications foams are deposited on porous substrates: hair, skin, textile, sponges and so on, however even the basics of the interaction of Newtonian and non-Newtonian foams with porous materials have never been thoroughly investigated: there is only one publication in the area where a complex interaction of foams with porous substrates was investigated [75,76].

It was found in $[75,76]$ that the kinetics of foam drainage on a porous substrate in the case of foam built up by a Newtonian liquid depends on three dimensionless numbers related to the properties of both foam and a porous substrate, and initial liquid volume fraction inside the foam. The result showed that there are three different regimes of the drainage process from foam [75,76]: (a) rate of imbibition into the porous substrate is faster than the rate of drainage from the foam; (b) a comparable rate of drainage and imbibition; (c) rate of imbibition is slower as compared with the rate of drainage. In the latter case, the liquid volume fraction at foam/porous substrate interface reaches maximum limiting value at the moment tm and a free liquid layer starts to form on the surface of the porous substrate. However, in the end, the free liquid layer is sucked by a porous substrate and it completely disappears at particular moment. After that moment again all liquid coming from the foam goes directly into the porous substrate and the liquid volume fraction decreases to its final value. The developed model $[75,76]$ can be used to model and predict the amount of active ingredients to the porous substrate (skin, hair).

\section{Acknowledgements}

This research was supported by European Science Foundation Marie Curie ITN grant CoWet; Engineering and Physical Research Council, UK grants EP/J010596/1 and EP/D077869/1; Procter \& Gamble; European Space Agency under grants FASES, PASTA and MAP EVAPORATION; COST MP1106 EU project. 



\section{Nomenclature}

\begin{tabular}{|c|c|}
\hline$A$ & Hamaker constant \\
\hline$a$ & Capillary length \\
\hline$D$ & distance between the fibres \\
\hline$D_{0}$ & initial wetted diameter of the droplet \\
\hline$h$ & film thickness, equilibrium liquid profile, droplet height \\
\hline$h_{s}$ & thickness of thin porous substrate \\
\hline$h^{*}$ & scale of the droplet height \\
\hline$H$ & drop height at the drop apex \\
\hline $\mathrm{Ht}$ & Blood's Haematocrit (\%) \\
\hline$K$ & flow behaviour index \\
\hline$K_{p}$ & permeability of the porous substrate \\
\hline$l$ & radius of the wetted area inside the porous substrate \\
\hline $\bar{l}$ & dimensionless radius of the wetted area inside the porous substrate \\
\hline$l^{*}$ & radius of wetted region at the end of the process \\
\hline$L$ & radius of droplet base \\
\hline$L_{a d}$ & maximum radius of droplet base in a partial wetting case \\
\hline$L_{m}$ & maximum radius of droplet base in a complete wetting case \\
\hline$L_{0}$ & initial radius of droplet base \\
\hline$L^{*}$ & scale of the droplet base \\
\hline $\bar{L}$ & dimensionless droplet base \\
\hline$m$ & Porosity of the porous substrate \\
\hline$N C M$ & nitrocellulose membrane \\
\hline$n$ & flow behavior index \\
\hline$p$ & vapor pressure over a curved interface \\
\hline$p_{a}$ & ambient air pressure \\
\hline$p_{c}$ & capillary pressure inside the pores of the porous layer \\
\hline$R$ & universal gas constant \\
\hline$R_{f}$ & radius of fibres \\
\hline RBCs & red blood cells \\
\hline$R H$ & relative humidity \\
\hline$r$ & radial co-ordinate \\
\hline$x$ & coordinate axis \\
\hline
\end{tabular}




$\begin{array}{ll}t & \text { time } \\ T & \text { temperature } \\ t_{c} & \text { Transitional time taken to go from capillary to gravitational spreading } \\ & \text { regime } \\ t_{h s} & \text { time taken for complete saturation of porous substrate in vertical direction } \\ t_{m} & \text { the time when radius of droplet base reaches to its maximum value, } L_{m} \\ t_{\mu}^{*} & \text { time scale for viscous spreading } \\ t_{p}^{*} & \text { time scale for imbibition into porous medium } \\ t_{0} & \text { duration of initial fast stage of spreading } \\ t^{*} & \text { time taken for complete imbibition } \\ \bar{t} & \text { dimensionless time } \\ u & \text { vertical velocity } \\ v & \text { radial velocity } \\ v_{m} & \text { molar volume the liquid } \\ V & \text { droplet volume } \\ V_{0} & \text { initial droplet volume } \\ V_{l} & \text { volume of liquid that can be accumulated within the bunch of hair } \\ V_{t o t} & \text { total volume of hair tresses } \\ W B C s & \text { white blood cells } \\ w & \text { Effective lubrication parameter }\end{array}$

\section{Greek Symbols}

porosity of hair tresses

$\dot{\gamma} \quad$ shear rate

$\gamma \quad$ surface tension

$\mu \quad$ viscosity

$\rho \quad$ density

$\theta \quad$ contact angle

$\theta_{\text {ad }} \quad$ advancing contact angle

$\theta_{f} \quad$ final contact angle

$\theta_{m} \quad$ contact angle at maximum droplet base radius

$\theta_{r} \quad$ receding contact angle 


\section{References}

[1] Oron A, Davis SH, Bankoff SG. Long-scale evolution of thin liquid films. Rev Mod Phys 1997;69:931-80. doi:10.1103/RevModPhys.69.931.

[2] Bonn D, Eggers J, Indekeu J, Meunier J. Wetting and spreading. Rev Mod Phys 2009;81:739_ 805. doi:10.1103/RevModPhys.81.739.

[3] Eral HB, 'T Mannetje DJCM, Oh JM. Contact angle hysteresis: A review of fundamentals and applications. Colloid Polym Sci 2013;291:247-60. doi:10.1007/s00396-012-2796-6.

[4] Attinger D, Moore C, Donaldson A, Jafari A, Stone HA. Fluid dynamics topics in bloodstain pattern analysis: Comparative review and research opportunities. Forensic Sci Int 2013;231:375-96. doi:10.1016/j.forsciint.2013.04.018.

[5] Brutin D. Droplet Wetting and Evaporation From Pure to Complex Fluids. Hayton, Joe; 2015.

[6] Yakhno TA, Sedova OA, Sanin AG, Pelyushenko AS. On the existence of regular structures in liquid human blood serum (plasma) and phase transitions in the course of its drying. Tech Phys 2003;48:399-403. doi:10.1134/1.1568479.

[7] Zhao Y, Jones SA, Brown MB. Dynamic foams in topical drug delivery What are topical foams? J Pharm Pharmacol 2010;62:678-84. doi:10.1211/jpp/62.06.0003.

[8] Young T. An Essay on the Cohesion of Fluids. Philos Trans R Soc London 1805;95:65-87. doi:10.1098/rstl.1805.0005.

[9] Cazabat, A. M., Cohen Stuart M a. Dynamics of Wettlng: Effects of Surface Roughness. J Phys Chem 1986;90:5845-9. doi:10.1021/j100280a075.

[10] Savva N, Kalliadasis S, Pavliotis GA. Two-dimensional droplet spreading over random topographical substrates. Phys Rev Lett 2010;104:1-4. doi:10.1103/PhysRevLett.104.084501.

[11] Vellingiri R, Savva N, Kalliadasis S. Droplet spreading on chemically heterogeneous substrates. Phys Rev E - Stat Nonlinear, Soft Matter Phys 2011;84:1-14. doi:10.1103/PhysRevE.84.036305.

[12] Starov, V. M.; Velarde, M. G.;Radke CJ. Wetting and Spreading Dynamics. CRC Press, Taylor \& Francis Group; 2007.

[13] De Gennes PG. Solvent evaporation of spin cast films:“crust” effects. Eur Phys J E Soft Matter Biol Phys 2002;7:31-4. doi:10.1140/epje/i200101169.

[14] Starov VM. Equilibrium and hysteresis contact angles. Adv Colloid Interface Sci 1992;39:147-73. doi:10.1016/0001-8686(92)80059-7.

[15] Schwartz LW, Eley RR. Simulation of Droplet Motion on Low-Energy and Heterogeneous Surfaces. J Colloid Interface Sci 1998;202:173-88. doi:10.1006/jcis.1998.5448.

[16] Tanner LH. The spreading of silicone oil drops on horizontal surfaces. J Phys D Appl Phys 1979;12:1473-84. doi:10.1088/0022-3727/12/9/009.

[17] Starov VM, Kalinin V V., Chen J Den. Spreading of liquid drops over dry surfaces. Adv 
Colloid Interface Sci 1994;50:187-221. doi:10.1016/0001-8686(94)80030-8.

[18] Chen J Den. Experiments on a spreading drop and its contact angle on a solid. J Colloid Interface Sci 1988;122:60-72. doi:10.1016/0021-9797(88)90287-1.

[19] Taniguchi M, Pieracci JP, Belfort G. Effect of undulations on surface energy: A quantitative assessment. Langmuir 2001;17:4312-5. doi:10.1021/la001791l.

[20] Starov VM, Kostvintsev SR, Sobolev VD, Velarde MG, Zhdanov SA. Spreading of Liquid Drops over Dry Porous Layers: Complete Wetting Case. J Colloid Interface Sci 2002;252:397-408. doi:10.1006/jcis.2002.8450.

[21] Alleborn N, Raszillier H. Spreading and sorption of droplets on layered porous substrates. J Colloid Interface Sci 2004;280:449-64. doi:10.1016/j.jcis.2004.08.003.

[22] Clarke A, Blake TD, Carruthers K, Woodward A. Spreading and imbibition of liquid droplets on porous surfaces. Langmuir 2002;18:2980-4. doi:10.1021/la0117810.

[23] Acton JM, Huppert HE, Worster MG. Two-dimensional viscous gravity currents flowing over a deep porous medium. J Fluid Mech 2001;440:359-80. doi:10.1017/S0022112001004700.

[24] Davis SH, Hocking LM. Spreading and imbibition of viscous liquid on a porous base. II. Phys Fluids 2000;12:1646-55. doi:10.1063/1.870416.

[25] Ahmed G, Sellier M. Modeling the Effects of Absorption on Spreading Dynamics. Transp Porous Media 2016;112:637-63. doi:10.1007/s11242-016-0668-0.

[26] Starov VM, Zhdanov SA, Velarde MG. Capillary imbibition of surfactant solutions in porous media and thin capillaries: Partial wetting case. J Colloid Interface Sci 2004;273:589-95. doi:10.1016/j.jcis.2004.02.033.

[27] Starov VM, Kostvintsev SR, Sobolev VD, Velarde MG, Zhdanov SA. Spreading of Liquid Drops over Saturated Porous Layers. J Colloid Interface Sci 2002;246:372-9. doi:10.1006/jcis.2002.8450.

[28] Replogle R., Meiselman H., Merrill E. Clinical implications of blood rheology studies. Circulation 1967;148.

[29] Morris C., Rucknagel D., Shukla R, Gruppo R., Smith C., Blackshear P. Evaluation of the yield stress of normal blood as a function of fibrinogen concentration and hematocrit. Microvasc Res 1989;323.

[30] Chao TC, Trybala A, Starov V, Das DB. Influence of haematocrit level on the kinetics of blood spreading on thin porous medium during dried blood spot sampling. Colloids Surfaces A Physicochem Eng Asp 2014;451:38-47. doi:10.1016/j.colsurfa.2014.03.033.

[31] Perktold K, Karner G, Leuprecht A, Hofer M. Influence of non-Newtonian Flow Behavior on Local Hemodynamics. J Appl Math Mech 1999;189.

[32] Hell K., Balzereit M, Diebold U, Bruhn H. Importance of blood viscoelasticity in arteriosclerosis. Angiology 1989;539.

[33] Merrill E. Rheology of Blood. Physiol Rev 1969;863.

[34] Thiriet M. Biology and Mechanics of Blood Flows Part II: Mechanics and Medical Aspects. Springer-Verlag New York; 2008.

[35] Brutin D, Sobac B, Loquet B, Sampol J. Pattern formation in drying drops of blood. J Fluid 
Mech 2011;667:85-95. doi:10.1017/S0022112010005070.

[36] Bou Zeid W, Brutin D. Influence of relative humidity on spreading, pattern formation and adhesion of a drying drop of whole blood. Colloids Surfaces A Physicochem Eng Asp 2013;430:1-7. doi:10.1016/j.colsurfa.2013.03.019.

[37] Chao TC, Arjmandi-Tash O, Das DB, Starov VM. Spreading of blood drops over dry porous substrate: Complete wetting case. J Colloid Interface Sci 2015;446:218-25. doi:10.1016/j.jcis.2015.01.054.

[38] Chao TC, Arjmandi-Tash O, Das DB, Starov VM. Simultaneous spreading and imbibition of blood droplets over porous substrates in the case of partial wetting. Colloids Surfaces A Physicochem Eng Asp 2016;505:9-17. doi:10.1016/j.colsurfa.2015.10.056.

[39] Merrill EW, Gilliland ER, Cokelet G, Shin H, Britten A, Wells, R. E. J. Rheology of blood and flow in the microcirculation. J Appl Physiol 1963;18:255-60.

[40] Bou-Zeid W, Brutin D. Effect of relative humidity on the spreading dynamics of sessile drops of blood. Colloids Surfaces A Physicochem Eng Asp 2014;456:273-85. doi:10.1016/j.colsurfa.2014.05.004.

[41] Carré A, Eustache F. Spreading kinetics of shear-thinning fluids in wetting and dewetting modes. Langmuir 2000;16:2936-41. doi:10.1021/la991021d.

[42] Rafaï S, Bonn D. Spreading of non-Newtonian fluids and surfactant solutions on solid surfaces. Phys A Stat Mech Its Appl 2005;358:58-67. doi:10.1016/j.physa.2005.06.017.

[43] Rafaï S, Sarker D, Bergeron V, Meunier J, Bonn D. Superspreading: Aqueous surfactant drops spreading on hydrophobic surfaces. Langmuir 2002;18:10486-8. doi:10.1021/la020271i.

[44] Sobac B, Brutin D. Desiccation of a sessile drop of blood: Cracks, folds formation and delamination. Colloids Surfaces A Physicochem Eng Asp 2014;448:34-44. doi:10.1016/j.colsurfa.2014.01.076.

[45] Laan N, Smith F, Nicloux C, Brutin D. Morphology of drying blood pools. Forensic Sci Int 2016;267:104-9. doi:10.1016/j.forsciint.2016.08.005.

[46] Brutin D, Sobac B, Nicloux C. Influence of Substrate Nature on the Evaporation of a Sessile Drop of Blood. J Heat Transfer 2012;134:61101. doi:10.1115/1.4006033.

[47] Sefiane K. Patterns from drying drops. Adv Colloid Interface Sci 2014;206:372-81. doi:10.1016/j.cis.2013.05.002.

[48] Sefiane K. On the formation of regular patterns from drying droplets and their potential use for bio-medical applications. J Bionic Eng 2010;7:S82-93. doi:10.1016/S1672-6529(09)60221-3.

[49] Chen R, Zhang L, Zang D, Shen W. Blood drop patterns: Formation and applications. Adv Colloid Interface Sci 2016;231:1-14. doi:10.1016/j.cis.2016.01.008.

[50] Rapis E. A change in the physical state of a nonequilibrium blood plasma protein film in patients with carcinoma. Tech Phys 2002;47:510-2. doi:10.1134/1.1470608.

[51] Buzoverya ME, Shcherbak YP, Shishpor I V. Quantitative estimation of the microstructural inhomogeneity of biological fluid facies. Tech Phys 2014;59:1550-5. doi:10.1134/S1063784214100119.

[52] Bremmer RH, De Bruin KG, Van Gemert MJC, Van Leeuwen TG, Aalders MCG. Forensic quest for age determination of bloodstains. Forensic Sci Int 2012;216:1-11. 
doi:10.1016/j.forsciint.2011.07.027.

[53] Feughelman M. Mechanical Properties of Alpha-Keratin Fibres: Wool, Human Hair and related properties. Univ.New South Wales Press; 1997.

[54] Robbins CR. Chemical and Physical Behavior of Human Hair. Springer; 2012.

[55] Zviak C. The Science of Hair Care. New York: Marcel Dekker; 1986.

[56] Dupres V, Langevin D, Guenoun P, Checco A, Luengo G, Leroy F. Wetting and electrical properties of the human hair surface: Delipidation observed at the nanoscale. J Colloid Interface Sci 2007;306.

[57] Yuan Y, Lee RT. Contact Angle and Wetting Properties. Surf Sci Tech Springer Ser Surf Sci 2013;51:3-34. doi:10.1007/978-3-642-34243-1.

[58] Lodge RA, Bhushan B. Wetting properties of human hair by means of dynamic contact angle measurement. J Appl Polym Sci 2006. doi:10.1002/app.24774.

[59] Kamath YK, Dansizer CJ, Weigmann H-D. Surface Wettability of Human Hair. I. Effect of Deposition of Polymers and Surfactants n.d.

[60] Kamath YK, Dansizer CJ, Weigmann H-D. Surface Wettability of Human Hair. II. Effect of Temperature on the Deposition of Polymers and Surfactants n.d.

[61] Trybala A, Bureiko A, Kovalchuk N, Arjmandi-Tash O, Liu Z, Starov V. Wetting properties of cosmetic polymeric solutions on hair tresses. Colloids Surfaces A 2015.

[62] Förster T, Schwuger MJ. Correlation between adsorption and the effects of surfactants and polymers on hair. Interfaces Condens Syst Prog Colloid Polym Sci 1990;83:104-9.

[63] Ran G, Zhang Y, Song Q, Wang Y, Cao D. The adsorption behavior of cationic surfactant onto human hair fibers. Colloids Surfaces B Biointerfaces 2009;68:106-10. doi:10.1016/j.colsurfb.2008.09.024.

[64] Bureiko A, Trybala A, Huang J, Kovalchuk N, Starov V. Bulk and surface rheology of Aculyn TM 22 and Aculyn TM 33 polymeric solutions and kinetics of foam drainage. Colloids Surfaces A Physicochem Eng Asp 2013;434:268-75. doi:10.1016/j.colsurfa.2013.05.072.

[65] Bureiko A, Trybala A, Huang J, Kovalchuk N, Starov V. Effects of additives on the foaming properties of Aculyn 22 and Aculyn 33 polymeric solutions. Colloids Surfaces A Physicochem Eng Asp 2014;460:265-71. doi:10.1016/j.colsurfa.2014.02.052.

[66] Keenan A, Haas RA. Hair gel formulations, useful for hair styling and as e.g. mousses, sprays and aerosols, contains Acudyne 180 hair fixative polymer thickened with combinations of Aculyn 22, 28, or 33 rheology modifiers to give improved formulation. Patent Number: RD478008-A, n.d.

[67] http://www.dow.com/assets/attachments/business/pcare/aculyn/aculyn_22/tds/aculyn22.pdf. Rohm Haas Copyr 2002.

[68] http://www.dow.com/assets/attachments/business/pcare/aculyn/aculyn_33/tds/aculyn33.pdf. Rohm Haas Copyr 2002.

[69] Bico J, Thiele U, Quéré D. Wetting of textured surfaces. Colloids Surfaces A Physicochem Eng Asp 2002;206:41-6. doi:10.1016/S0927-7757(02)00061-4.

[70] Bormashenko E, Musin A, Whyman G, Zinigrad M. Wetting transitions and depinning of the 
triple line. Langmuir 2012;28:3460-4. doi:10.1021/la204424n.

[71] Barbieri L, Wagner E, Hoffmann P. Water wetting transition parameters of perfluorinated substrates with periodically distributed flat-top microscale obstacles. Langmuir 2007;23:172334. doi:10.1021/la0617964.

[72] Wang J, Chen D. Criteria for entrapped gas under a drop on an ultrahydrophobic surface. Langmuir 2008;24:10174-80. doi:10.1021/la801092y.

[73] Arzhavitina A, Steckel H. Foams for pharmaceutical and cosmetic application. Int J Pharm 2010;394:1-17. doi:10.1016/j.ijpharm.2010.04.028.

[74] Arjmandi-Tash O, Trybala A, Mahdi FM, Kovalchuk NM, Starov V. Foams built up by nonNewtonian polymeric solutions: Free drainage. Colloids Surfaces A Physicochem Eng Asp 2016. doi:10.1016/j.colsurfa.2016.07.097.

[75] Bureiko A, Arjmandi-Tash O, Kovalchuk N, Trybala A, Starov V. Interaction of foam with a porous medium: Theory and calculations. Eur Phys J Spec Top 2015;224:459-71. doi:10.1140/epjst/e2015-02374-2.

[76] Arjmandi-Tash O, Kovalchuk N, Trybala A, Starov V. Foam drainage placed on a porous substrate. Soft Matter 2015;11:3643-52. doi:10.1039/C5SM00377F. 